\title{
Rüdiger Graf
}

\section{Die Mentalisierung des Nirgendwo und die Transformation der Gesellschaft}

\author{
Der theoretische Utopiediskurs in Deutschland 1900-1933
}

Im Unterschied zu den übrigen Beiträgen des Bandes, die konkrete Formen und Funktionen utopischen Denkens im Europa in der Zwischenkriegszeit zum Gegenstand haben, wird im folgenden der von Wissenschaftlern und Intellektuellen geführte theoretische Diskurs über das Phänomen der Utopien bzw. des Utopischen analysiert. Die Untersuchung dieses Diskurses, der in Deutschland im 19. Jahrhundert einsetzte und bis $\mathbf{1 9 3 3}$ seine noch heute charakteristischen Ausprägungen erhielt ${ }^{1}$, geschieht mit der Absicht, die Virulenz des konkreten utopischen Denkens über seine Reflexion in der zeitgenössischen Utopietheoriebildung zu erfassen. Zunächst waren in theoretischen Studien, die das Phänomen der Utopie in grundsätzlicher Weise zu erfassen gesucht hatten, unter dem Begriff "Utopie ${ }^{\star}$ die Spielarten der an Thomas Morus' Schrift "De optimo rei publicae statu, deque nova insula Utopia“ (1516) anschließenden Literaturgattung - die sogenannten „Staatsromane“ - subsumiert worden. Im übertragenen und alltagssprachlichen Sinn bezeichnete „Utopie ${ }^{\alpha}$ einen unausführbaren Weltverbesserungsplan, der von einem Schwärmer, i.e. einem „Utopisten“, formuliert wurde, so daß der Begriff häufig in pejorativer und diffamierender Weise verwendet wurde ${ }^{2}$. In Auseinandersetzung mit dem literaturgeschichtlichen Utopiediskurs

1 Vgl. zur gegenwärtigen Utopieforschung und dem Bezug auf den Utopiediskurs der 20er Jahre: Richard Saage, Zum Stand der sozialwissenschaftlichen Utopieforschung I-III, in: Neue politische Literatur 38, $39,40(1993,1994,1995)$ 221-238, 55-97 u. 441-451; im folgenden zitiert: Saage, Zum Stand; Richard Saage, Utopieforschung. Eine Bilanz (Darmstadt 1997); Wilhelm Vosskamp (Hrsg.), Utopieforschung. Interdisziplinäre Studien zur neuzeitlichen Utopie, 3 Bde. (Stuttgart 1982); im folgenden zitiert: Vosskamp, Utopieforschung. Sowie exemplarisch für die Geschichtswissenschaft: Otto Gerbard Oexle, Wunschräume und Wunschzeiten. Entstehung und Funktionen des utopischen Denkens in Mittelalter, Früher Neuzeit und Moderne, in: Die Wahrheit des Nirgendwo: Zur Geschichte und Zukunft des utopischen Denkens, hrsg. v. Jörg Calließ (Rehburg-Loccum 1994) 33-83, v.a. 34-39; im folgenden zitiert: Calließ, Wahrheit des Nirgendwo.

2 Brockhaus' Konversations-Lexikon, Bd. 16 (14. volist. neubearb. Aufl., Berlin, Wien 1895) 146: „Utopien (Utopia), nach dem Griechischen soviel als Nirgendswo, nannte der englische Kanzler Thomas More (s.o.) die fabelhafte Insel, auf welcher er seinen Staatsroman ,De op- 
sowie dem pejorativen Alltagsverständnis etablierte sich im ersten Drittel des 20. Jahrhunderts in Deutschland ein Utopieverständnis, das am pointiertesten Karl Mannheim nach seiner Emigration 1934 in der „Encyclopaedia of the Social Sciences“ zusammenfaßte: Die Utopie, so definierte Mannheim, ist erstens eine Bewußtseinsform ( ${ }_{n}$ mental outlook ${ }^{\text {) }}$, die sich an seinstranszendenten Vorstellungen orientiert; sie verursacht - zweitens - kollektive Handlungen, die darauf abzielen, die Realität in Richtung auf diese seinstranszendenten Vorstellungen zu verändern ${ }^{3}$. Diese beiden Annahmen, die Utopie sei eine Bewußtseinsform und spiele eine zentrale Rolle in gesellschaftlichen Transformationsprozessen, konstituierten ein Utopieverständnis, das im folgenden als "sozialpsychologisch“ bezeichnet wird. Im vorliegenden Aufsatz soll genauer geklärt werden, wie sich dieses Verständnis, das den Utopiebegriff zur sozialwissenschaftlichen Analyse nutzbar machte, herausbildete und welche Kontexte zu seiner Etablierung beitrugen.

Dazu ist es zunächst erforderlich, den zugrunde gelegten Diskursbegriff kurz zu erläutern. Historische Arbeiten, die den Diskursbegriff analytisch fruchtbar machen, versuchen entweder in der Tradition Michel Foucaults, die unpersönliche Ordnung zu rekonstruieren, welche die Produktion von Texten kanalisiert, organisiert und kontrolliert ${ }^{4}$; oder sie verstehen den Diskurs im Sinne der anglo-amerikanischen Sprachphilosophie als das Produkt von Sprecherinnen und Sprechern in bestimmten Handlungszusammenhängen ${ }^{5}$. Ich schließe an die zweite Tradition und verstehe zunächst - mit Jürgen Habermas - als „Diskurs“ die „... durch Argumentation gekennzeichnete Form der Kommunikation, in der problematisch gewordene Geltungsansprüche zum Thema gemacht und auf ihre Berechtigung

timo rei publicae statu, deque nova insula Utopia' (Löwen 1516 u.ö.) spielen ließ. Nach dieser Schrift wurde die ganze Gattung von Schriften U. genannt, deren Eigentümlichkeit darin besteht, daß der Verfasser die von ihm gewünschten Änderungen der Rechtsordnung als bereits durchgeführt voraussetzt und die Wirkungen dieser Änderungen am Bilde eines erdichteten Staatswesens zeigt. Statt U. nennt man solche Schriften auch Staatsromane (s.o.). In neuester Zeit bezeichnet man Socialreformer, welche den Boden der Wirklichkeit verlassen und sich mit phantastischen Weltverbesserungsplänen beschäftigen, als Utopisten." Ähnlich definierte zuvor bereits: Staats- und Gesellschaftlexikon, hrsg. v. Herrmann Wagener, Bd. 19 (Berlin 1866) 126f. Wie der Brockhaus dann auch: Meyers Großes Konversationslexikon, Bd. 19 (6. gänzlich neubearb. u. verm. Aufl., Leipzig, Wien 1909) 982. Zur Geschichte des Utopiebegriffs: Lucian Hölscher, Utopie, in: Geschichtliche Grundbegriffe. Lexikon zur politisch-sozialen Sprache in Deutschland, hrsg. v. Otto Brunner, Werner Conze, Reinhart Koselleck, Bd. 6 (Stuttgart 1996) 733-788; im folgenden zitiert: Hölscher, Utopie.

${ }^{3} \mathrm{Karl}$ Mannheim, Utopia, in: Encyclopaedia of the Social Sciences, Bd. 15 (London 1934) 200-203, bes. 201; im folgenden zitiert: Mannheim, Utopia.

$+\mathrm{Vgl}$. die Behandlung des Begriffs der Ordnung in Michel Foucault, Die Ordnung der Dinge. Eine Archäologie der Humanwissenschaften (Frankfurt a. M. ${ }^{13} 1995$ ) 22-25; sowie ders., Die Ordnung des Diskurses (Frankfurt a.M. 1991) 9f. und passim.

5 Zur Unterscheidung der Diskursbegriffe: John E. Toews, Intellectual History after the Linguistic Turn. The Autonomy of Meaning and the Irreducibility of Experience, in: AHR 92 (1987) 879-907, hier: 890; Günther Lottes, „The State of the Art“. Stand und Perspektiven der nintellectual history “, in: Neue Wege der Ideengeschichte. Festschrift für Kurt Kluxen zum 85. Geburtstag, hrsg. v. Frank-Lothar Kroll (Paderborn u. a. 1996) 27-45; Peter Schöttler, Wer hat Angst vor dem „linguistic turn“" , in: GG 23 (1997) 134-151, hier: $138 \mathrm{f}$. 
hin untersucht werden "6. Diese Definition mißt den Diskursteilnehmenden mit ihren Meinungen, Wünschen und Interessen zentrale Bedeutung für die Gestaltung des Diskurses zu. Die Annahme, der Diskurs werde grundsätzlich von den Teilnehmenden gestaltet, impliziert jedoch nicht, diese seien dabei vollkommen autonom. Vielmehr weist jeder historisch vorzufindende Diskurs eine bestimmte Grundstruktur auf, die man auf der Basis von Donald Davidsons Sprach- und Interpretationstheorie als Set geteilter Grundannahmen verstehen kann: Damit Kommunikation über ein Thema überhaupt zustande kommen kann, müssen Sprechende und Interpretierende über eine große Menge gleicher Meinungen über die gemeinsame Welt verfügen, weil erst vor diesem Hintergrund Differenzen und Meinungsunterschiede über bestimmte Themen entstehen können ${ }^{7}$. Diese den Diskurs strukturierenden Grundannahmen - im folgenden: die Diskursformation - sind nicht vollständig in die Verfügung der Teilnehmenden gestellt, da sie in einer gegebenen Diskurssituation nicht immer problematisiert werden können, aber sie sind prinzipiell explizierbar und somit veränderlich ${ }^{8}$. Ein solcher Diskursbegriff hat gegenüber statischeren Diskursmodellen im Stile Foucaults den Vorzug, daß er die Analyse von Diskurstransformationen und des Beitrages, den die Diskursteilnehmenden an ihnen haben, erlaubt ${ }^{9}$. Da er zudem den Sprecherinnen und Sprechern keine vollkommene Autonomie einräumt, sondern die Wirkung von inner- und außerdiskursiven Kontextfaktoren integrieren kann, ermöglicht er die Analyse und Erklärung der Herausbildung des sozialpsychologischen Utopiediskurses in idealer Weise.

In der gegenwärtigen Theoriediskussion der intellectual history wird das Problem des Kontextualismus zumeist auf die Frage reduziert, ob es möglich ist, einen Text unabhängig von seinen Kontexten zu verstehen ${ }^{10}$. Jenseits des bloßen Textverständnisses gibt es jedoch weitere Fragen an Texte oder Diskurse, die nur

6 Jürgen Habermas, Wahrheitstheorien (1972), in: Vorstudien und Ergänzungen zur Theorie des kommunikativen Handelns (Frankfurt a. M. 1995) 127-186, hier: 131.

7 Als Einführung zur Philosophie Donald Davidsons siehe: Donald Davidson, Davidson, Donald, in: A Companion to the Philosophy of Mind, hrsg. v. Samuel Guttenplan (Oxford 31996) 231-236; detaillierter: Donald Davidson, Radical Interpretation, in: Inquiries into Truth and Interpretation (Oxford 1973) 125-139, bes. 137; sowie Donald Davidson, On the Very Idea of a Conceptual Scheme (1974), in: ebd. 183-198, bes. 197.

8 Damit unterscheidet sich dieser Begriff "Diskursformation" von dem Michel Foucaults, der darunter die Systeme von Regelmäßigkeiten und Streuungen bestimmter Elemente in den Aussagen der untersuchten Texte versteht. $\mathrm{Vgl}$.: Michel Foucault, Die Archäologie des Wissens (Frankfurt a. M. ${ }^{8} 1997$ ) 48-60, bes. 58.

9 Das Fehlen dieser Möglichkeit, wenn man dem Foucaultschen Diskursbegriff folgt, wird inzwischen immer häufiger als Mangel begriffen. Vgl.: Ian Maclean, The Process of Intellectual Change. A Post-Foucauldian Hypothesis, in: Cultural History after Foucault, hrsg. v. John Neubauer (Berlin, New York 1999) 163-176, 166: „... Foucault could not elaborate a theory of intellectual change ..."

10 Die These des Kontextualismus, daß dies nicht möglich sei, wird kritisiert von Mark Bevir, The Errors of Linguistic Contextualism, in: History and Theory 31 (1992) 276-298; vgl. auch John Patrick Diggins, The Oyster and the Pearl. The Problem of Contextualism in Intellectual History, in: History and Theory 23 (1984) 151-169. 
über Kontextanalysen beantwortet werden können, nämlich beispielsweise warum etwas zu einem bestimmten Zeitpunkt auf eine bestimmte Weise formuliert wurde und nicht etwas anderes in anderer Form. Um die Etablierung der sozialpsychologischen Diskursformation nicht nur darzustellen, sondern auch Ansätze zu ihrer Erklärung zu liefern, müssen also die Kontexte spezifiziert werden, die ihre Herausbildung beeinflußten ${ }^{11}$. Wenn man von einem teilnehmerbasierten Diskursbegriff ausgeht, kann die Relevanz der ausgewählten Kontexte dadurch bestimmt werden, daß ihre Wirkung auf die Diskursteilnehmenden ausgewiesen wird. Dabei darf jedoch das Explikationsverhältnis von Text und Kontext nicht einfach vorausgesetzt oder gar der Text auf einen Kontext reduziert werden, sondern ihre Beziehung ist selbst immer wieder zu problematisieren ${ }^{12}$.

In der Forschung herrscht weitgehender Konsens darüber, daß sich zu Beginn des 20. Jahrhunderts eine wesentliche Veränderung des theoretischen Utopiediskurses vollzog. Diese Veränderung wird jedoch auf verschiedene Weise beschrieben: von Lucian Hölscher als beginnende positive Verwendung des Begriffs, in deren Folge die Utopien zum anerkannten Medium der Zukunftsreflexion wurden ${ }^{13}$, von Bernhard Braun als beginnende soziologische Verwendung ${ }^{14}$ und von Richard Saage als Dynamisierung bzw. beginnende intentionale Verwendung des Utopiebegriffs ${ }^{15}$. Im folgenden schließe ich an Lucian Hölschers Artikel aus den "Geschichtlichen Grundbegriffen" an, der die "geschichtsphilosophische Einbindung" des Utopiebegriffs als ein zentrales Element der sozialpsychologischen Diskursformation bestimmt ${ }^{16}$. Darüber hinaus wird jedoch der Mentalisierung des Utopiebegriffs größere Bedeutung zugemessen, d.h. dem Prozeß, in dessen Verlauf "Utopie" nicht mehr als Staatsroman, sondern zunächst als Ausdruck einer bestimmten Bewußtseinsform und dann als diese Bewußtseinsform selbst

11 Der Begriff „Kontext “ stammt aus der Sprachwissenschaft und bezeichnet die sprachliche oder nicht-sprachliche Umgebung eines Ausdrucks, insofern sie für dessen Bedeutung relevant ist. Vgl. U. M. Quasthoff, Context, in: The Encyclopedia of Language and Linguistics, Bd. 2 (Oxford u. a. 1994) 730-737, bes. 730 f. Was für einen bestimmten Diskurs ein relevanter Kontext ist, kann nicht allgemein, sondern über konkrete Plausibilitätsargumente bestimmt werden.

12 Vgl.: Dominick LaCapra, Rethinking Intellectual History and Reading Texts, in: Modern European Intellectual History. Reappraisals and New Perspectives, hrsg. v. Dominick LaCapra, Steven Kaplan (Ithaca, London 1982) 47-85, bes. 49, 57, 82.

${ }^{13}$ Hölscher, Utopie 752.

14 Bernhard Braun, Die Siedlung. Der Beginn des Sozialismus. Gesellschaftskritik, Siedlung, Utopie bei Gustav Landauer, in: Gustav Landauer im Gespräch, hrsg. v. Hanna Delf, Gert Mattenklott (Tübingen 1997) 191-201, hier: 199; im folgenden zitiert: Braun, Die Siedlung; Delf, Mattenklott, Landauer im Gespräch.

15 Richard Saage, Begriffe, Gestalten und Kontexte utopischen Denkens, in: Calließ, Wahrheit des Nirgendwo 15-31, hier: 16 f. So bereits zuvor: Bernhard Braun, Die Utopie des Geistes. Zur Funktion der Utopie in der politischen Theorie Gustav Landauers ( $W_{1 s s}$. Schriften Reihe 11, Beiträge zur Philosophie 103, Idstein 1991) 10-12; im folgenden zitiert: Braun, Utopie.

16 Hölscher, Utopie 775-788. Hölschers für das 19. Jahrhundert hervorragende begriffsgeschichtliche Darstellung bleibt - der Anlage des Lexikons gemäß - für das 20. Jahrhundert sehr knapp. 
verstanden wurde. Erst diese Mentalisierung des Begriffs wertete die Utopie in ihrer historischen Rolle auf und machte sie für die sozialwissenschaftliche Analyse gesellschaftlicher Transformationsprozesse fruchtbar.

Die Unklarheit darüber, was genau das Neuartige des Utopiediskurses ausmacht, führt auch zu unterschiedlichen Datierungen seiner Entstehung. Ernst Blochs eigene Einflußnahme auf die Darstellung des Utopiediskurses hat vermutlich dazu beigetragen, daß er selbst zum Urheber des modernen Utopiebegriffs erklärt wurde ${ }^{17}$. Demgegenüber haben Arno Münster und Bernhard Braun herausgearbeitet, daß Blochs Utopietheorie wesentlich auf Gustav Landauers Arbeiten basiert. Daher setzen Braun und Saage die Entstehung eines neuen oder veränderten Utopiediskurses bereits bei ihm an ${ }^{18}$. Darüber hinaus weist Lucian Hölscher Ansätze einer geschichtsphilosophischen Einbindung der Utopie bereits im 19. Jahrhundert nach ${ }^{19}$. Auf der Basis einer inhaltlichen und rezeptionsgeschichtlichen Analyse der Beiträge zum theoretischen Utopiediskurs werde ich im ersten Teil des Aufsatzes argumentieren, daß die Etablierung der sozialpsychologischen Diskursformation mit Andreas Voigts und Gustav Landauers Studien 1906 bzw. 1907 begann und mit der Rezeption der Schriften von Karl Mannheim und Hans Freyer am Ende der Weimarer Republik abgeschlossen war. In der Kontextanalyse im zweiten Teil soll dann gezeigt werden, daß die Herausbildung der sozialpsychologischen Formation des Utopiediskurses als ein Versuch von Sozialwissenschaftlern und Intellektuellen zu verstehen ist, vor dem Hintergrund intensiver eigener Krisenerfahrung gesellschaftliche Transformationsprozesse zu erfassen und sich selbst in diesen Prozessen eine zentrale Rolle als Beobachtende und Handelnde zuzuweisen.

17 Bereits in den 20er Jahren war Bloch darum bemüht zu verbreiten, die Auffassung der Utopie als Bewußtseinsform sei seine Erfindung. $V_{g l}$. Blochs Reaktion auf Karl Mannheims "Ideologie und Utopie" in seinem Brief an Siegfried Kracauer vom 30. 4. 1929, in: Ernst Bloch, Briefe 1903-1975, hrsg. v. Karola Bloch u. a., 2 Bde. (Frankfurt a. M. 1985) hier: Bd. 1 313: "Schon das Wort Utopie im Buchsinn und als Gegensatz zu Ideologie ist nicht auf Mannheims Boden gewachsen. Utopie bedeutete bislang Staatsroman, hatte jedenfalls nicht den metaphysischen Sinn, den ihm Mannheim jetzt geben kann. ,Seinstranszendente Vorstellungen, die die Welt verwandeln, auch sprengen" - gar: ,die aus der Welt hinaustretenden Spannungen werden zum Sprengstoff in der Welt' $\ldots$ diese Definitionen, ja der ganze Terminus, utopisches Bewußtsein " wären ohne mein Buch (Der Geist der Utopie, R.G.) wohl kaum möglich. " Im folgenden zitiert: Bloch, Briefe. Siehe auch: Emst Bloch, Erbschaft dieser Zeit. Erweiterte Ausgabe (Frankfurt a. M. 1985) 288. Noch Gert Ueding erklärt, Bloch habe den Begriff der Utopie "und die von ihın gemeinte Sache aus seiner ursprünglichen, eingeschränkten Bedeutung einer romanhaften Staats- und Sozialutopie befreit" ${ }^{4}$. Gert Ueding, Ernst Blochs Philosophie der Utopie, in: Vosskamp, Utopieforschung 1 (Stuttgart 1982) 293-303, hier: 293. So auch Wilhelm Kamlah, Utopie, Eschatologie, Geschichtsteleologie. Kritische Untersuchungen zum Ursprung und zum futurischen Denken der Neuzeit (Mannheim, Wien, Zürich 1969) 13; und Theodor W. Adorno, in: Gespräche mit Ernst Bloch, hrsg. v. Rainer Traub, Harald Wieser (Frankfurt a.M. 1977) 58, im folgenden zitiert: Traub, Wieser, Gespräche.

18 Amo Münster, Utopie, Messianismus und Apokalypse im Frühwerk von Ernst Bloch (Frankfurt 2. M. 1982) 124-126; im folgenden zitiert: Münster, Utopie; vgl. auch Braun, Die Utopie 10-12; und im Anschluß an ihn Saage, Zum Stand I 222.

19 Hölscher, Utopie 775-785. 


\section{Vom Phantasiegebilde zur seinsverändernden Kraft - der theoretische Utopiediskurs}

\section{Die literaturgeschichtliche Diskursformation}

In der zweiten Hälfte des 19. Jahrhunderts wurde der theoretische Utopiediskurs vorwiegend von Staatswissenschaftlern und Nationalökonomen geführt, die sich in quasi literaturgeschichtlichen Abhandlungen der Gattung der Staatsromane widmeten. Dabei beabsichtigten sie zumeist, entweder Inspirationen für die eigene Arbeit zu gewinnen oder eine bestimmte Art der Gesellschaftsbetrachtung zu diskreditieren. In Deutschland begann die Gattungsgeschichtsschreibung mit Robert v. Mohl (1799-1875). Seine Definition der Utopien als Staatsromane, das heißt als „Dichtungen ..., welche die Schilderung eines idealen Gesellschafts- oder Staatslebens zum Gegenstande haben, sei es nun, daß dabei die Form einer Reisebeschreibung, einer statistischen Schilderung oder einer Lebensgeschichte benutzt wurde ${ }^{20}$, blieb für den literaturgeschichtlichen Utopiediskurs bis in das 20. Jahrhundert hinein prägend ${ }^{21}$. In Reaktion auf den zahlenmäßigen Anstieg literarischer Utopien nahmen in den $1890 \mathrm{er}$ Jahren auch die literaturgeschichtlichen Abhandlungen in einem solchen Ausmaß zu22, daß Carl Grünberg 1905 in seiner Rezension von Eugen Heinrich Schmitts Buch "Der Idealstaat ${ }^{“}$ urteilte, man habe es "mit einer jener überflüssigen und wertlosen literarischen Erscheinungen zu tun, mit denen in jüngster Zeit der Markt immer häufiger überschwemmt" werde ${ }^{23}$.

Erste Ansätze einer Mentalisierung des Utopiediskurses stellten die Versuche einiger Autoren dar, die literarischen Utopien zu einer bestimmten Fähigkeit des Bewußtseins in Beziehung zu setzen. So erklärte beispielsweise der Jurist Arthur

20 Robert v. Mohl, Die Staatsromane. Ein Beitrag zur Literaturgeschichte der Staatswissenschaften, in: Zeitschrift für die gesamte Staatswissenschaft 2 (1845) 24-74, 27; im folgenden zitiert: v. Mobl, Die Staatsromane. Vgl. zur Gattungsgeschichtsschreibung in internationaler Perspektive: Hölscher, Utopie 746-752.

21 In diesem Sinn definieren z. B.: Friedrich v. Kleinwächter, Die Staatsromane. Ein Beitrag zur Lehre vom Communismus und Socialismus (Wien 1891) 19; im folgenden zitiert: Kleinwächter, Die Staatsromane; Arthur v. Kirchenbeim, Schlaraffia politica. Geschichte der Dichtungen vom besten Staate (Leipzig 1892) $1 \mathrm{f}$.; im folgenden zitiert: $v$. Kirchenheim, Schlaraffia Politica; Rudolf Stammler, Utopien, in: Deutsche Rundschau 70 (1892) 281-296, 291 f.; Friedrich Fürst v. Wrede, Die Entwicklung des Staatsromanes, in: Deutsche Revue 30 (1905) 141-156, hier: 156; Julius Reiner, Berühmte Utopisten und ihr Staatsideal. Plato, Morus, Campanella, Cabet (Jena 1906) 1, 6; im folgenden zitiert: Reiner, Berühmte Utopisten; Joseph Prys, Der Staatsroman des 16. und 17. Jahrhunderts (Diss. Würzburg 1913) 1 f.; Edgar Salin, Platon und die griechische Utopie (München 1921) V.

22 Diese Kausalität wurde bereits von den Zeitgenossen konstruiert. Vgl.: Kleinwächter, Die Staatsromane 4 und $v$. Kirchenheim, Schlaraffia Politica $1 \mathrm{f}$.

${ }^{23}$ Carl Grünberg, Dr. Eugen Heinrich Schmitt: Der Idealstaat, in: Zeitschrift für Socialwissenschaft 8 (1905) 66-67, hier: 67; im folgenden zitiert: Schmitt, Der Idealstaat. Die Zunahme der Utopiegeschichten wurde bereits bemerkt von: Hölscher, Utopie 749 und Richard Toby Widdicombe, Early Histories of Utopian Thought (to 1950), in: Utopian Studies 3 (1992) 1-38, hier: 2. 
v. Kirchenheim (1855-1924) die Utopie zu einem Produkt der menschlichen Phantasie, weshalb die Geschichte der Utopien gleichzeitig Auskunft gebe über die "Geschichte der menschlichen Sehnsucht nach Glück, nach Frieden "24. Die ersten Reflexionen über das Verhältnis von Utopie und Geschichte folgten ebenso dem literaturgeschichtlichen Paradigma, so daß vor allem danach gefragt wurde, ob eine Utopie, i.e. ein Staatsroman, Aufschluß über eine bestimmte gesellschaftliche Konstellation geben und ob sie gesellschaftsverändernde Kraft entfalten könne. Für Theodor Mundt (1808-1861) waren die Utopien ein Indikator dafür, $\mathrm{da} \beta_{n}$ ein heimliches Ziehen und Zucken durch die Gesellschaft (hingehe), und daß unheilvolle Bewegungen auf dem Grunde derselben sich entzünden “ könnten ${ }^{25}$. Diesem Befund stimmte der Philosoph Ludwig Stein (1859-1930) zu, indem er die Utopisten als die „poetischen Sturmvögel “ beschrieb, „die das orkanartige Heranrauschen einer neuen Zeit ${ }^{\text {“ }}$ verkündeten ${ }^{26}$. Die Utopie selbst bzw. der Staatsroman wurde dabei jedoch als Epiphänomen mit keiner oder nur sehr geringer Wirkung angesehen. Denn Bücher, so argumentierte Friedrich v. Kleinwächter (1838-1927), könnten die Welt nicht verändern, sondern sie hätten - ausgehend von den gebildeten Kreisen, in denen sie gelesen würden - allenfalls eine allmähliche Wirkung auf menschliche Gedankenwelten ${ }^{27}$.

\section{Die mentalistische Wende und die geschichtsphilosophische Einbindung}

Die Konzeptionalisierung des Verhältnisses von Utopie und Geschichte veränderte sich in dem Maße, in dem der Utopiebegriff mentalisiert wurde, i.e. in dem die Utopie als Ausdruck einer Bewußtseinsform bzw. selbst als Bewußtseinsform verstanden wurde. Von zentraler Bedeutung für diese mentalistische Wende des Utopiediskurses war die 1906 publizierte Vortragsreihe über „Die sozialen Utopien“ des Frankfurter Mathematikers und Ökonomen Andreas Voigt (1860-1941) ${ }^{28}$. Im Unterschied zu den vorher publizierten Geschichten der Staatsromane wollte Voigt mit seinen vor dem Freien Deutschen Hochstift gehal-

24 v. Kirchenheim, Schlaraffia Politica 6. Ebenso, wenn auch mit deutlich negativerer Konnotation: Emil Döll, Das Schicksal aller Utopien oder socialen Charlatanerien und das verstandesgemäß Reformatorische (Leipzig 1897) 1: „...diese Phantasie kann schöpferisch sein, aber: oft bewegt sie sich in zügellosen, oft recht kindischen Ausschweifungen und führt in dieser entarteten Gestalt zu jenen wüsten Gebilden, deren Conception und Erzeugung man Phantastik nennt."

25 Theodor Mundt, Die Geschichte der Gesellschaft in ihren neueren Entwickelungen und Problemen (Leipzig 1844) 304.

26 Ludwig Stein, Die soziale Frage im Lichte der Philosophie (Stuttgart 1897) 290.

27 Kleinwächter, Die Staatsromane 149. In diesem Sinne urteilten auch: $v$. Mobl, Die Staatsromane 25 und 61 f.; $v$. Kirchenheim, Schlaraffia Politica 278 und positiver: Reiner, Berühmte Utopisten 74: „Man kann ohne Übertreibung sagen, daß die Utopien den Boden für die sozialen Reformen vorbereitet haben."

${ }^{28}$ Andreas Voigt, Die sozialen Utopien - Fünf Vorträge (Leipzig 1906); im folgenden zitiert: Voigt, Utopien. In den bisherigen Darstellungen der Geschichte des Utopiediskurses wird Andreas Voigt zumeist gar nicht behandelt. Zumindest erwähnt wird er in einer Aufzählung bei: Hölscher, Utopie 786. 
tenen Vorträgen über die Geschichte der Utopien „ein Stück Weltanschauungslehre" leisten. Das hielt er für möglich, weil sich in den Utopien als holistischen Entwürfen erstrebenswerter Welten die Bewußtseinslage derer ausdrücke, die an sie glauben ${ }^{29}$. Mit der Behandlung der Utopien als Ausdruck individueller oder kollektiver Bewußtseinsformen wurden Utopien nicht mehr als die literarischen Produkte einzelner verstanden, und damit erhielt die Reflexion auf die Rolle der Utopien in der Geschichte eine sozialpsychologische Fundierung. Utopien spielten bei Voigt eine reale historische Rolle, weil der Glaube an sie "Menschen erfassen und in ihrem politischen Handeln bestimmen" könne ${ }^{30}$.

Unmittelbar nach der Publikation wurde Andreas Voigts Vortragsreihe intensiv und breit rezipiert ${ }^{31}$. Die Rezensenten begriffen seine Absicht, nan einigen Beispielen die Psychologie des Utopismus klar(zu)machen ${ }^{42}$, als Neuerung und begrüßten sie mehrheitlich ${ }^{33}$. Obwohl Voigts Ausführungen anti-utopistisch und sozialismusfeindlich waren, wurde seine Grundidee, kollektive Mentalitäten über Utopien zu erschließen, auch im linken politischen Spektrum positiv aufgenommen. So schloß Hugo Nathanson in seiner Rezension im "Blaubuch" an die Mentalisierung des Utopiebegriffs an und betonte die historische Bedeutung der Utopien noch stärker als Voigt: ${ }_{n}$ Was gestern noch phantastische Utopie eines einsamen Geistes gewesen, wird heute vielleicht ein ernstlich und mit zäher Ausdauer verfolgtes Ziel großer gesellschaftlicher Gruppen, kann morgen schon gesetzlich geheiligte Ordnung von angeblich ewiger Dauer werden. ${ }^{\text {} 34}$

Man kann begründeterweise vermuten, daß der Anarchist und Literat Gustav Landauer (1870-1919) Andreas Voigts Utopiekonzeption durch diese Rezension

29 Voigt, Utopien 6 und 22: „Man kann mit Recht sagen, daß sie (die Utopie, R.G.) alle Seiten des menschlichen Geistes und menschlichen Lebens berührt, und daß die Utopien in ihrer Weise ein vollständiges Spiegelbild menschlichen Lebens und Strebens darbieten. "Hölscher, Utopie 786, lokalisiert die Anfänge der Betrachtung der Utopie als Ausdruck kollektiver Mentalitäten bereits bei Schmitt, Der Idealstaat und Reiner, Berühmte Utopisten. Hier handelt es sich jedoch um bloße Erklärungen, die nicht analytisch wirksam werden. Darüber hinaus war Voigts Studie einflußreicher als die anderen.

30 Voigt, Utopien 1.

31 So finden sich mindestens elf Rezensionen in Zeitschriften mit unterschiedlicher wissenschaftlicher und politischer Ausrichtung: Kritische Blätter für die gesamten Sozialwissenschaften (1906), Das Blaubuch. Wochenschrift für öffentliches Leben, Literatur und Kunst (1906), Deutscher Kampf (1906), Sociale Praxis. Zentralblatt für Sozialpolitik (1906), Philosophische Wochenschrift (1906), Die Grenzboten. Zeitschrift für Literatur und Kunst (1907), Literarisches Centralblatt für Deutschland (1908), Der Alte Glaube. Literarische Beilage (1908), Philosophisches Jahrbuch der Görres-Gesellschaft (1908), Jahrbücher für Nationalökonomie und Statistik (1908) und Archiv für Kulturgeschichte (1909).

32 Anonymus, Utopismus und soziale Praxis, in: Die Grenzboten 66 (1907) 50-52, hier: 50.

33 Vgl. insbesondere: Paul Dienstag, Theoretische Sozialökonomie, in: Kritische Blätter für die gesamten Sozialwissenschaften 2 (1906) 578-580; Leopold v. Wiese, Prof. Dr. Andreas Voigt, Die sozialen Utopien, in: Sociale Praxis. Zentralblatt für Sozialpolitik 15 (1906) Sp. 1261 f.; und Fr. Botbe, A. Voigt, Die sozialen Utopien, Leipzig 1906, in: Archiv für Kulturgeschichte 7 (1909) $366 \mathrm{f}$.

34 Hugo Nathanson, Voigt, Die sozialen Utopien, in: Das Blaubuch 1 (1906) 1443-45, hier: 1443. 
im „Blaubuch“ kennenlernte, bevor er sich selbst dem Thema Utopie widmete ${ }^{35}$. In seinem Buch „Die Revolution“, das 1907 in Martin Bubers Reihe „Die Gesellschaft. Sammlung sozialpsychologischer Schriften " und damit an einem zentralen Publikationsort soziologisch orientierter Wissenschaftler im Kaiserreich erschien $^{36}$, ging Landauer allerdings über Voigt hinaus. Er verstand die Utopie selbst als ein geistiges Prinzip, das sich im individuellen oder kollektiven Bewußtsein ausprägen könne. Auf der Basis dieses mentalisierten Utopiebegriffs entwikkelte Landauer ein explizites und theoretisches Modell der Rolle der Utopien in der Geschichte. Die relativ stabilen Ordnungen des gesellschaftlichen, politischen und wirtschaftlichen Lebens bezeichnete Landauer als "Topien“ und erklärte, diese würden durch Utopien verflüssigt und verändert ${ }^{37}$. Utopien seien zunächst „ein Gemenge individueller Bestrebungen und Willenstendenzen, die immer heterogen und einzeln vorhanden sind". Aber sie könnten sich "in einem Moment der Krise ... durch die Form begeisterten Rausches zu einer Gesamtheit und zu einer Mitlebensform vereinigen und organisieren “38. Im Anschluß an diese revolutionären Prozesse werde jedoch nie die Utopie, sondern immer nur eine neue Topie realisiert ${ }^{39}$.

Wenngleich die unmittelbare Wirkung von Landauers Studie gering war ${ }^{40}$, entwickelte sie doch mittel- und langfristig großen Einfluß auf den theoretischen Utopiediskurs. Dieser dürfte wesentlich auf Landauers Engagement in der Münchner Räterepublik sowie seine Zugehörigkeit zum Spektrum linker und jüdischer Intellektueller - und hier insbesondere seiner Freundschaft mit Martin

35 Diese Vermutung ist gerechtfertigt, weil der Herausgeber des Blaubuchs zu dem Zeitpunkt, als die Rezension veröffentlicht wurde (20.9. 1906) Gustav Landauer zur Mitarbeit an der Zeitschrift überreden wollte, und Landauer bereits plante, ein Buch zum Thema Revolution zu verfassen. Vgl. den Brief Gustav Landauers an Fritz Mauthner vom 29.10. 1906 in: Gustav Landauer - Fritz Mauthner. Briefwechsel 1890-1919, bearb. v. Hanna Delf (München 1994) 150; und den Brief von Martin Buber an Gustav Landauer vom Juli 1906, in: Martin Buber, Briefwechsel aus sieben Jahrzehnten, Bd. 1: 1897-1918, hrsg. v. Grete Schaeder (Heidelberg 1972) 245.

36 Gustav Landauer, Die Revolution (Frankfurt a. M. 1907); im folgenden zitiert: Landauer, Revolution. Zur Schriftenreihe, in der u. a. Werner Sombart, Fritz Mauthner, Willy Hellpach, Franz Oppenheimer, Eduard Bernstein, Hellmuth v. Gerlach und Ferdinand Tönnies publizierten, siehe: Paul Mendes-Flobr, Von der Mystik zum Dialog. Martin Bubers geistige Entwicklung bis hin zu, Ich und Du' (Königstein/Taunus 1978) 111-130.

37 Landauer, Revolution 12.

38 Ebd.

39 Ebd. 14.

40 Die IBR verzeichnet für das Buch nur zwei Rezensionen, in denen zudem eher Landauers Utopie als sein Beitrag zum theoretischen Utopiediskurs thematisiert wurde: S. Saenger, Die Revolution, in: Die neue Rundschau 19 (1908) 922-923; und $J$. L., Die Revolution von Gustav Landauer, in: Ethische Kultur 16 (1908) 103. Die überraschend geringe unmittelbare Wirkung stellt ebenso fest: Christine Holste, „Die grausigste Ideenlosigkeit in ihren Dienst zwingen." Gustav Landauers Entwicklung zum utopischen Denken, in: Gustav Landauer. Eine Bestandsaufnahme zur Rezeption seines Werkes, hrsg. v. Leonhard M. Fiedler u.a. (Frankfurt a. M., New York 1995) 98-117, hier: 104. 
Buber - beruhen ${ }^{41}$. Trotz fehlender Zitate kann speziell für Ernst Bloch nachgewiesen werden, daß seine Utopiekonzeption in hohem Maße von der Lektüre von Gustav Landauers „Die Revolution ${ }^{\alpha}$ beeinflußt war ${ }^{\wedge 2}$. Darüber hinaus war Landauer ein zentraler Bezugspunkt für Leopold v. Wieses und Karl Mannheims Versuche, sich den Themen Revolution und Utopie zu nähern ${ }^{43}$. Unabhängig von Gustav Landauer wirkte auch Andreas Voigts Konzept der Weltanschauungsanalyse auf den Utopiediskurs der zwanziger Jahre44. Die Schriften von Voigt und Landauer stellten also die Wende zur sozialpsychologischen Formation des Utopiediskurses dar, die durch die Mentalisierung des Utopiebegriffs und seine geschichtsphilosophische Einbindung gekennzeichnet war.

\section{Die Etablierung der sozialpsychologischen Diskursformation in der Weimarer Republik}

Nach dem Ersten Weltkrieg intensivierte sich der theoretische Utopiediskurs in drei verschiedenen intellektuellen Spektren: erstens im Spektrum linker, zu groBen Teilen jüdischer Intellektueller, zweitens im Spektrum der liberalen Sozialwissenschaftler und drittens im Spektrum der sogenannten „Konservativen Revolution ${ }^{45}$. Diese Diskursspektren kennzeichneten jeweils spezifische intellektuelle Traditionen und enge persönliche und geistige Verbindungen, so daß sie je verschiedene Thematisierungskontexte für den Utopiediskurs bildeten. Nichtsdesto-

41 Zur Position Landauers im Spektrum jüdischer Intellektueller: Michael Löwy, Der romantische Messianismus Gustav Landauers, in: Delf, Mattenklott, Landauer im Gespräch 91-104; Michael Löwy, Erlösung und Utopie. Jüdischer Messianismus und libertäres Denken. Eine Wahlverwandtschaft (Berlin 1997) bes. 174-188; im folgenden zitiert: Löwy, Erlösung; Christine Holste, Der Forte-Kreis (1910-1915). Rekonstruktion eines utopischen Versuchs (Stuttgart 1992) 200; im folgenden zitiert: Holste, Forte-Kreis. Über die Beziehung zu Buber: Wermer Licharz, Gustav Landauer und Martin Buber in Briefen und Briefdialogen, in: Martin Buber (1878-1965). Symposium zum 20. Todestag, hrsg. v. Werner Licharz, Heinz Schmidt, Bd. 2 (Frankfurt a. M. 1989) 178-202, bes. 192.

42 Im Anschluß an Anton Christen zeigt Arno Münster in überzeugender Weise, daß Bloch nsich im Geist der Utopie und im Thomas Münzer ... an von Landauer vorgeprägte Begriffe, Definitionen und Auffassungen stark anlehnt...“, Münster, Utopie 125.

43 Vgl. Leopold $v$. Wiese, Die Problematik einer Soziologie der Revolution, in: Verhandlungen des Dritten Deutschen Soziologentages vom 24. und 25. September 1922 in Jena (Tübingen 1923) 6-23; Karl Mannheim, Ideologie und Utopie (Frankfurt a.M. ${ }^{41965, ~ ' 1929) ~ 173 f . ; ~}$ im folgenden zitiert: Mannbeim, Ideologie.

44 Zitate u. a. bei: Hans Girsberger, Der utopische Sozialismus des 18. Jahrhunderts in Frankreich und seine philosophischen und materiellen Grundlagen (Zürcher Volkswirtschaftliche Forschungen 1, Zürich 1924) 3; Siegfried Landshut, Utopisten, in: Religion in Geschichte und Gegenwart, Bd. 5 (Tübingen 21931); Mannheim, Utopia 203. Auch außerhalb des deutschen Sprachraumes wurde Voigt rezipiert: Joyce Oramel Hertzler, The History of Utopian Thought (New York 1923) 263.

45 Dieser Begriff ist genauso wie die Einteilung des politischen-intellektuellen Spektrums der Weimarer Republik in links und rechts wiederholt kritisiert worden. Da die Klassifikationen hier nicht zur exakten Bezeichnung inhaltlicher Positionen, sondern zur Unterscheidung intellektueller Milieus dienen, erscheint die Verwendung trotzdem gerechtfertigt. 
weniger entwickelte sich in allen Spektren überraschenderweise die gleiche sozialpsychologische Diskursformation, was im folgenden zunächst dargestellt und dann erklärt werden soll.

Auf der politischen Linken war der sogenannte "Utopismus" seit Karl Marx" und Friedrich Engels' Auseinandersetzung mit dem utopischen Sozialismus und ihrer Betonung der Wissenschaftlichkeit der eigenen Anschauungen verpönt und wurde in Diskussionen immer wieder als Vorwurf formulierrt6. Insbesondere innerhalb der deutschen Sozialdemokratie führte Karl Kautsky (1854-1938) im Zuge der Pragmatisierung sozialdemokratischer Politik einen regelrechten Feldzug gegen alle utopistischen Abweichler vom orthodoxen Marxismus ${ }^{47}$. Gegen diese Ausblendung des utopischen Elements aus der sozialistischen Politik wandten sich zu Beginn des 20. Jahrhunderts junge, linke und vornehmlich jüdische Intellektuelle. Sie traten für Utopien ein und propagierten einen Utopiebegriff, demzufolge die Utopie als Handlungsleitbild eine zentrale Rolle in historischen Transformationsprozessen spielte ${ }^{48}$. So entwickelte Gustav Landauer nicht nur die bereits dargestellte Utopietheorie, sondern auch eine Utopie dezentraler Siedlungsgenossenschaften ${ }^{49}$. Mit beiden erzielte er - nicht zuletzt durch sein Engagement in der Münchener Räterepublik - breite Wirkung im linksintellekruellen Milieu. Als Leiter des Zentralwirtschaftsamtes in der Räterepublik mit Gustav Landauer bekannt geworden, vertrat auch Otto Neurath (1882-1945) utopische Vorstellungen einer ${ }_{n}$ Vollsozialisierung ${ }^{*}$ der Wirtschaft ${ }^{50}$. Gemeinsam mit Wolfgang Schu-

$46 \mathrm{Vgl}$. als klassischen Text Friedrich Engels, Die Entwicklung des Sozialismus von der

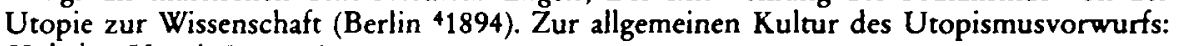
Hölscher, Utopie 755-768.

47 So Lucian Hölscher, Weltgericht oder Revolution. Protestantische und sozialistische Zukunftsvorstellungen im deutschen Kaiserreich (Stuttgart 1989) 318-322. Vgl. die Position Kautskys z. B. in: Karl Kautsky, Vorläufer des neueren Sozialismus, Bd. 3: Die beiden ersten großen Utopisten (Stuttgart, Berlin 21922) bes. 56-60.

48 Dies führte nicht zwangsläufig zu einer Ablehnung des Marxismus, sondern konnte wie bei Bloch als Wiederentdecken der wahren Marxschen Tradition angesehen werden: „Die Theorie der Utopie findet sich schon bei Marx. Nicht gerade in einem dogmatisch verstandenen Marxismus, aber bei Marx ist sie durchaus da. Das Utopische, das ist das Werdende, die politische Zukunftsvision. Ich habe diesen Utopiebegriff lediglich vom marxistischen Standpunkt aus weiterentwickelt. “ Zitiert nach Amo Münster, Tagträume vom aufrechten Gang. Sechs Interviews mit Ernst Bloch (Frankfurt a.M. 1978) 111; im folgenden zitiert: Münster, Tagträume.

$49 \mathrm{Zu}$ Landauers utopischen Konzeptionen und seinem politischen Engagement siehe: Eugene Lunn, Prophet of Community. The Romantic Socialism of Gustav Landauer (Berkeley, Los Angeles, London 1973) bes. 172-200; Ruth Link-Salinger, Gustav Landauer. Philosopher of Utopia (Indianapolis 1977) bes. 51-81; Holste, Forte-Kreis 179-241.

so Dazu: Hans-Joachim Dahms, Michael Neumann, Sozialwissenschaftler in der Münchener Räterepublik, in: Jahrbuch für Soziologiegeschichte (1992) 115-146, bes. 122-126; Nancy Cartwright u. a., Otto Neurath. Philosophy Between Science and Politics (Cambridge 1996) 43-53 und passim; allgemein zu Otto Neurath: Paul Neurath, Otto Neurath (1882-1945) Life and Work, in: Encyclopedia and Utopia. The Life and Work of Otto Neurath (1882-1945), hrsg. v. Elisabeth Nemeth, Friedrich Stadler (Dordrecht, Boston, London 1996) 15-28. 
mann (1878-1964) definierte er die Utopie - in Analogie zur Ingenieursarbeit - als "gesellschaftstechnische Konstruktion“, i.e. als Vorarbeit für die zukünftige Gesellschaftsgestaltung und forderte die Einrichtung einer wissenschaftlichen Utopistik an den Schulen und Universitäten ${ }^{51}$. Nach einer geistigen Erneuerung des Sozialismus strebten zudem religiöse Sozialisten wie Paul Tillich, der der Utopie oder dem Utopischen ebenfalls große Handlungsrelevanz zusprach ${ }^{52}$.

Die Etablierung des sozialpsychologischen Utopieverständnisses wurde auf der politischen Linken jedoch insbesondere vorangetrieben durch das intellektuelle Spektrum um Georg Lukács (1885-1971), Walter Benjamin (1892-1940) und Ernst Bloch (1885-1977), der zu beiden zeitweilig in einem engen, nsymbiotischen" Freundschaftsverhältnis stand ${ }^{53}$. Wenngleich Lukács sich später von seinen messianisch-utopischen Zielsetzungen distanzierte ${ }^{54}$, hatte er doch vor der Publikation von „Geschichte und Klassenbewusstsein “ (1923) gemeinsam mit Ernst Bloch eine Position der revolutionären Romantik oder des revolutionären Messianismus eingenommen 55 .

51 Mit der kurzzeitigen Herausgabe einer "Wirtschaft und Lebensordnung " betitelten Beilage zur Zeitschrift „Der Kunstwart“ sowie der Leitung des 1917 gegründeten Kriegswirtschaftsmuseums in Leipzig versuchten Neurath und Schumann, der Verwirklichung ihrer Ideen näher zu kommen. Vgl. Otto Neurath, Die Utopie als gesellschaftstechnische Konstruktion, in: Durch die Kriegswirtschaft zur Naturalwirtschaft (München 1919) 228-231; im folgenden zitiert: Neurath, Die Utopie; Otto Neurath, Utopien, in: Wirtschaft und Lebensordnung. Beilage zu Der Kunstwart 15 (1919) 5f.; Wolfgang Schumann, Utopien und Utopistik, in: Der Kunstwart 32 (1919) 4-10.

52 Vgl.: Paul Tillich, Kairos. Zur Geisteslage und Geisteswendung (Darmstadt 1926) 10: „Der Gedanke an die tatsächlichen Grenzen, die jedes Kommende haben muß, ist im Handeln selbst nicht wirksam und darf es nicht sein. Darum enthält alles auf Umgestaltung der Gegenwart gerichtete, unbedingt entschlossene Handeln Geist der Utopie."

${ }^{3} \mathrm{Vgl}$. Blochs eigene Beschreibungen der Freundschaften im Gespräch mit Iring Fetscher und Georg Lukács, in: Traub, Wieser, Gespräche 32f. und Münster, Tagträume 48. Allgemeiner zur Konstitution der Gruppe und zu ihrem Interesse an Utopie, Eschatologie und Messianismus: Löwy, Erlösung 36-39; Anson Rabinbach, Between Enlightenment and Apocalypse. Benjamin, Bloch and Modern Jewish Messianism, in: New German Critique 34 (1985) 78-124; John Ely, Intellectual Friendship and Elective Affinities of Critical Theory, in: South Atlantic Quarterly 97 (1998) 187-224, 188 und passim; Paul Mendes-Flohr, "To Brush History Against the Grain ${ }^{\alpha}$. The Eschatology of the Frankfurt School and Ernst Bloch, in: Journal of the American Academy of Religion 51 (1983) 631-650, hier: 633.

54 Vgl. das Vorwort zu „Geschichte und Klassenbewusstsein“ von 1967; Georg Lukács, Geschichte und Klassenbewusstsein. Studien über marxistische Dialektik (Darmstadt, Neuwied 1970) 11-13 (1. Aufl. 1923).

55 Als anschauliche Schilderungen der messianisch-utopischen Stimmung von Bloch und Lukács in Heidelberg: Marianne Weber, Max Weber. Ein Lebensbild (Tübingen 1926) 474, 476; und Paul Honigsheim, Max Weber in Heidelberg, in: Max Weber zum Gedächtnis, hrsg. v. René König, Johannes Winckelmann (Kölner Zeitschrift für Soziologie und Sozialpsychologie, Sonderheft 7, Köln, Opladen 1963) 161-271, $187 \mathrm{f}$. Zu Georg Lukács' früher Position: Michael Löwy, Die revolutionäre Romantik von Bloch und Lukács, in: Verdinglichung und Utopie. Ernst Bloch und Georg Lukács zum 100. Geburtstag, hrsg. v. Amo Münster, Michael Löwy, Nicolas Tertulian (Frankfurt a.M. 1987) 17-29; Paul Breines, Utopie und Partei. Anmerkungen zum jungen Lukács, in: Deutsches utopisches Denken im 20. Jahrhundert, hrsg. v. Reinhold Grimm, Jost Hermand (Stuttgart u. a. 1974) 96-103. 
Innerhalb dieses Diskurszusammenhanges auf der politischen Linken entwikkelte Bloch seine Utopiekonzeption, die er zuerst ausführlich in seinem 1915-1917 entstandenen expressionistischen Frühwerk "Geist der Utopie ${ }^{4}$ darlegte ${ }^{56}$. Bloch verstand die Utopie - vollkommen losgelöst von den Staatsromanen - als das geistige Prinzip des "Noch-Nicht", als die dem Menschen innewohnende Idee einer idealen, erlösten Welt, die zu verwirklichen sei ${ }^{57}$. Dieses „Noch-Nicht “ bzw. das Utopische erhob er zur anthropologischen Konstante und erklärte es zum eigentlichen Movens in historischen Transformationsprozessen ${ }^{58}$. Das Grundanliegen, „das ,System des theoretischen Messianismus' sturmreif zu machen, für die Propheten, für den praktischen Messianismus " 59 , verfolgte Ernst Bloch mit seiner historischen Studie über Thomas Müntzer weiter ${ }^{60}$. Dort führte er die Gedanken, die er zuvor im "Geist der Utopie" theoretisch entwickelt hatte, nochmals an cinem Beispiel aus, indem er den sozialwissenschaftlichen Utopiebegriff zur Analyse der sozial-religiösen Bewegung um Thomas Müntzer nutzte. Gegen die Tradition der marxistischen Historiographie vertrat er die These, es sei das utopische Element, das Menschen zu kollektivem, gesellschaftsveränderndem Handeln bewege, und dieses müsse daher in der Analyse adäquat berücksichtigt werden: „Dergestalt also reicht die rein ökonomische Betrachtung nicht aus, um allein nur den Eintritt eines historischen Ereignisses von der Wucht des Bauernkrieges vollkommen, restlos konditional oder kausal zu erklären, geschweige denn, daß ihre Analyse imstande wäre, die tieferen Inhalte der hier aufglühenden Menschengeschichte und gar erst die encheiresis gloriae, das umgehende Prinzip des dritten Reiches aufzulösen, herabzustürzen, seines absolut originären Charakters zu entkleiden, zu reflexivieren und ins rein Ideologische zu entrealisieren. ${ }^{* 61}$

${ }^{56}$ Ernst Bloch, Geist der Utopie (Frankfurt a. M. 1959, Faksimile der Erstausgabe von 1918); im folgenden zitiert: Bloch, Geist (1918).

57 Vgl. Bloch, Geist (1918) 9. Später formulierte Bloch über das Anliegen des „Geist der Utopic ${ }^{\prime}:$.... das Stichwort ,Utopie‘ war nun gesetzt. Das hat nichts zu tun mit dem negativen Sinn der Utopie oder nur insofern zu tun, als Utopie kein Wischiwaschi ist, sondern geprüft sein muß, ausgeglichen mit der Welt, belehrt von der Welt, wie ich später sagte: docta spes, gelehrte Hoffnung und berichtigte Hoffnung - dies klang schon an. Aber die Hauptsache war erst, dieses Wort aus seinem herabsetzenden $\operatorname{Sinn}, \mathbf{N a}$, das ist ja bloß eine Utopie zu befreien und die ungeheure Vergangenheit der Utopie in der Tradition zu zeigen: Zukunft in der unabgegoltenen Vergangenheit auf allen möglichen Gebieten ...", in: Traub, Wieser, Gespräche 35.

58 Emst Bloch, Geist der Utopie (Berlin 1923) 4f., 209, $311,341$.

59 Bloch, Geist (1918) 337. Die Abfassung eines Systems des theoretischen Messianismus war zum damaligen Zeitpunkt Blochs Ziel. Vgl. Margarete Susman, Der Geist der Utopie, in: Frankfurter Zeitung (12.1. 1919).

60 Ernst Bloch, Thomas Münzer als Theologe der Revolution (München 1921). Im folgenden zitiert: Bloch, Münzer.

61 Bloch, Münzer 74. Und auch 128: „Und hat auch Marx den Anstoß zum neuen Leben mit Recht entscheidend auf den homo oeconomicus, auf die Beherrschung der ökonomischen Interessenpunkte gestellt, damit die allzu arkadisch vermutete hinterweltliche Paradiesesordnung des rationalen, im Kern chiliastischen Sozialismus hart und mit weltklugem Kampf gegen die Welt erobert werde: so stirbt man doch nicht für ein bloßes durchorganisiertes Pro- 
Bloch zieht in seiner Arbeit über Thomas Müntzer immer wieder Parallelen zwischen der Zeit Müntzers und der revolutionären Situation der Gegenwart ${ }^{62}$. Aufgrund dieses offenkundigen Präsentismus urteilten die Rezensenten übereinstimmend, das Buch sei weniger eine historische Studie als vielmehr ein zeitgenössisches Manifest, mit dem Bloch die utopischen Potentiale der Gegenwart stärken wolle ${ }^{63}$. Dieser Deutung lag die Erkenntnis zugrunde, daß Bloch das Utopische als zentrales, geistiges Movens in historischen Transformationsprozessen auffaßte. Insofern trug Ernst Bloch zur Popularisierung des sozialpsychologischen Utopieverständnisses - insbesondere auf der politischen Linken - bei.

Im Spektrum der akademischen Sozialwissenschaften etablierte insbesondere Karl Mannheim (1893-1947) die sozialpsychologische Formation des Utopiediskurses. Dieser war ebenfalls lange Zeit mit Georg Lukács befreundet, aber er vertrat deutlich liberalere Auffassungen ${ }^{64}$. Mannheim machte die Begriffe ${ }_{n}$ Ideologie" und "Utopie" zum basalen Analysevokabular in seiner Konzeption einer Wissenssoziologie, die das Ziel hatte, das Denken in seiner Seinsverbundenheit zu

duktionsbudget, so kehrt doch gerade im bolschewistischen Vollzug des Marxismus der alte gotteskämpferische, der taboritisch-kommunistisch-joachimitische Typus des radikalen Täufertums erkennbar wieder; mit einem noch verdeckten, geheimen Mythos des Wozu, als dessen Vorspiel und Korrektiv jedoch der Chiliasmus dauernd figuriert.

62 Bereits in der Ankündigung des Buches hieß es, Bloch behandle "überhaupt eine der unsrigen allernächst verwandte Zeit ${ }^{*}$ und stelle sie , in die weiterwirkenden, geschichtsphilosophischen Zusammenhänge". Zitat: Der neue Merkur 5 (1921/1922) 408; siehe auch: Bloch, Münzer 150f: „Aber andere, Münzer verwandte Tage sind wieder gekommen, und sie werden nicht ruhen, bis ihre Tat getan ist ... Nun stehen, großgewachsen, die Erben der Münzerschen Webergesellen und Tuchknappen auf dem revolutionären Plan, nicht mehr zu vertreiben ..." Und weiter heißt es: „Aber strahlend erscheint uns daran Thomas Münzer in Bild und Absicht wieder, Liebknecht mannigfach verwandt, als unerbirtlicher Organisator deutlich genug, um selbst Lenin, Napoleon des Kollektivismus, nicht fernzustehen ...".

${ }^{63} \mathrm{H}$. Hermelink, Ernst Bloch, Thomas Münzer als Theologe der Revolution, in: Theologische Blätter 2 (1923) 298; Franz Strunz, Thomas Münzer als Theologe der Revolution, in: Das literarische Echo 25 (1923) 751 f.; Alfons Paquet, Ein Theologe der Revolution, in: Der neue Merkur 6 (1922) 383-384; Walther Koch, Wiedertäufer, in: Sozialistische Monatshefte 69 (1923) 187-189. Vgl. stellvertretend Siegfried Kracauer, Prophetentum, in: Frankfurter Zeitung (27. 8. 1922): „... der Chiliast Bloch will vielmehr in der Gestalt Münzers Selbstbegegnung feiern, in Form voraussetzungsloser und uneigentlicher Geschichtsbetrachtung will er Blick und Gesinnung des heutigen Menschen auf das ,utopische Ende' und seine Verwirklichung lenken."

6t Zur intellektuellen Biographie Mannheims siehe: David Kettler, Volker Meja, Karl Mannheim and the Crisis of Liberalism. The Secret of these New Times (New Brunswick 1995); im folgenden zitiert: Kettler, Meja: Karl Mannheim; Colin Loader, The intellectual Development of Karl Mannheim. Culture, Politics, and Planning (Cambridge u. a. 1985); im folgenden zitiert: Loader, Karl Mannheim; Henk E. Woldring, Karl Mannheim. The Development of his Thought. Philosophy, Sociology and Social Ethics (van Gorcum 1986); im folgenden zitiert: Woldring, Karl Mannheim. Speziell zur Beziehung von Mannheim und Lukács: Éva Karádi, Erzsébet Vézer (Hrsg.), Georg Lukacs, Karl Mannheim und der Sonntagskreis (Frankfurt a. M. 1985); im folgenden zitiert: Karádi, Vézer, Sonntagskreis. Lee Congdon, Exile and Social Thought. Hungarian Intellectuals in Germany and Austria 1919-1933 (Princeton N.J. 1991) bes. 5-40, 266-296; im folgenden zitiert: Congdon, Exile. 
begreifen ${ }^{65}$. In expliziter Ablehnung "historisch-unkonstruktiver" Begriffsbildungen, die den Utopiebegriff auf Thomas Morus und die Gattung der Staatsromane einzuengen suchten, entwickelte Mannheim einen nsystematischen" Utopiebegriffot. Demzufolge sind Utopien diejenigen seinstranszendenten Vorstellungen. die das gesamte Bewußtsein beherrschen, so daß „Erlebnisform, Aktionsform, betrachrungsweise (Sicht) von hier aus sich organisieren " und die, , in das Handehn übergehend, die jeweils bestehende Seinsordnung zugleich teilweise oder ganz $z^{4}$ sprengen ${ }^{67}$. In seiner Darstellung der Entwicklung der geistigen und sozialen Bewegungen in der Neuzeit nutzte Mannheim den Begriff der Utopie, weil "die wesentlichsten Wandlungen der geistigen Struktur von der Transformation des Utopischen her zu fassen" seien $^{68}$. Auf diese Weise entwickelte er ein Phasennodell vom orgiastischen Chiliasmus der Wiedertäufer über die liberalhumantäre Idee und die konservative Idee zur sozialistisch-kommunistischen Utopie und der gegenwärtigen Konstellation ${ }^{69}$. Systematisch und inhaltlich griff Mannheim dabei - insbesondere in bezug auf Chiliasmus und Kommunismus auf die Studien von Fritz Gerlich, Ladislaus Radványi und Alfred Doren ${ }^{70}$ zurück, de die Wirkungsmacht des utopischen Bewußtseins in der Geschichte untersuchr hatten.

Die große unmittelbare Wirkung von "Ideologie und Utopie“ in Deutschland bis $1933^{71}$ hatte vor allem zwei Gründe. Zum einen war Mannheim seit seinem

65 Zur Vissenssoziologie in Deutschland: David Frisby, The Alienated Mind. The Sociology of Knovledge in Germany 1918-1933 (London 1992); zu Mannheim insbesondere 107-174; im folgenden zitiert: Frisby, Mind.

66 Ebd. : 75-177.

67 Ebd. i82, 169.

68 Ebd. 224.

${ }^{69}$ Ebd. : 84-225.

70 Alfrel Doren, Wunschräume und Wunschzeiten (Vorträge 1924/25 der Bibliothek Warburg, Lejpzig, Berlin 1927); Ladislaus Radványi, Der Chiliasmus. Ein Versuch zur Erkenntnis der chiliastischen Idee und des chiliastischen Handelns (Masch. Diss., Heidelberg 1923, Neuauşabe: Budapest 1985); Fritz Gerlich, Der Kommunismus als Lehre vom tausendjährigen Reich (München 1920). Gerlichs Studie kann hier zudem als - vielleicht einflußreichstes - Beispisl für ein ganzes Genre von Texten dienen, in denen zu Beginn der 20er Jahre versucht wurde, den Kommunismus als religiöse Bewegung zu deuten.

71 Bis 1933 erschienen 28 Rezensionen zum Teil mehrmals u. a. in a) Tageszeitungen: Berliner Tageblat (9.6. 1930), Frankfurter Zeitung (28.4. 1929), Kölnische Zeitung (27. 10. 1929), Vossische Zeitung (13. 2. 1930); b) sozialwissenschaftlichen Zeitschriften: Zeitschrift für Politik (19:9), Archiv für angewandte Soziologie (1929), Kölner Vierteljahrshefte für Soziologie (1929, 1431), Zeitschrift für Völkerpsychologie und Soziologie (1929), Archiv für systematische Phiosophie und Soziologie (1929), Zeitschrift für angewandte Psychologie und Charakterkunde (1930), Archiv für Sozialwissenschaft und Sozialpolitik (1930), Zeitschrift für Nationaökonomie (1931); c) politischen Zeitschriften: Die Gesellschaft (1929f.), Unter dem Banner ies Marxismus (1930), Deutsche Literaturzeitung (1930), Archiv für die Geschichte des Sozinlismus und der Arbeiterbewegung (1930), Der Kampf (1930), Freie Volksbildung (1931), Die Internationale (1931). Zur längerfristigen Rezeption siehe: Volker Meja, Nico Stehr (Hsg.), Der Streit um die Wissenssoziologie, Bd. 2: Rezeption und Kritik der Wissenssoziologe (Frankfurt a. M. 1982); im folgenden zitiert: Meja, Stebr, Der Streit. 
Auftritt auf dem Soziologentag von $1928^{72}$ der "shooting star ${ }^{\text {" der deutschen }}$ Soziologie und zum anderen hatte er engen Kontakt zum linksintellektuellen Spektrum, das er mit der Neuformulierung des Ideologiebegriffs direkt herausforderte ${ }^{73}$. Erwartungsgemäß kritisierten die vom marxistischen Standpunkt argumentierenden Rezensenten vornehmlich Mannheims Ausweitung des marxistischen Ideologiebegriffs auf alle gesellschaftlichen Standpunkte. So erklärte Adalbert Fogarasi, aus dem bürgerlichen Klassenstandpunkt folge die Falschheit von Meinungen (Ideologie), während der proletarische ihre Richtigkeit und damit die Möglichkeit zur gesellschaftsverändernden Utopie impliziere ${ }^{74}$. Diese Kritik wurde im Kern von Hans Speier, Otto Neurath, Herbert Marcuse, Max Horkheimer und Karl August Wittfogel geteilt ${ }^{75}$. Sowohl in diesen negativen Kritiken als auch in den positiven Rezensionen wie beispielsweise von Siegfried Kracauer, Günther Stern (später Günther Anders) und Michael Freund wurde jedoch Mannheims grundsätzliche Konzeption der Utopie als Bewußtseinsform, die historische Transformationsprozesse bewirkt, nicht in Frage gestellt ${ }^{76}$. Lediglich

72 Vgl. den Vortrag: Karl Mannheim, Die Bedeutung der Konkurrenz im Gebiete des Geistigen, in: Verhandlungen des Sechsten Deutschen Soziologentages vom 17. bis 19. September 1928 in Zürich (Tübingen 1929) 35-83 und die Reaktionen 84-115. Zu Mannheims Erfolg: Dirk Käsler, Der Streit um die Bestimmung der Soziologie auf den deutschen Soziologentagen 1910-1930, in: Soziologie in Deutschland und Österreich 1918-1945, hrsg. v. M. Rainer Lepsius (Opladen 1981) 199-244, bes. 227-232.

73 Dementsprechend liest sich auch die Liste der Rezensenten und Rezensentinnen mit Adalbert Fogarasi, Max Horkheimer, Siegfried Kracauer, Herbert Marcuse, Otto Neurath, Paul Tillich und Karl August Wittfogel wie das ,Who Is Who" des linksintellektuellen Spektrums der Weimarer Republik.

74 Adalbert Fogarasi, Die Soziologie der Intelligenz und die Intelligenz der Soziologie. (Beiträge) zur Theorie der Ideologie, in: Unter dem Banner des Marxismus 4 (1930) 359-375, hier: 364.

75 Hans Speier, Soziologie oder Ideologie? Bemerkungen zur Soziologie der Intelligenz, in: Die Gesellschaft 7 (1930) 357-372 (wieder abgedruckt in: Meja, Stebr, Der Streit 532-550); Otto Neurath, Bürgerlicher Marxismus, in: Der Kampf 23 (1930) 227-232 (wieder abgedruckt in: Meja, Stehr, Der Streit 584-593); Max Horkheimer, Ein neuer Ideologiebegriff?, in: Archiv für die Geschichte des Sozialismus und der Arbeiterbewegung 15 (1930) 33-56; Herbert Marcuse, Zur Wahrheitsproblematik der soziologischen Methode, in: Die Gesellschaft 2 (1929) 356-369 (wieder abgedruckt in: Meja, Stebr. Der Streit 459-473); Karl August Wittfogel, Wissen und Gesellschaft. Neuere deutsche Literatur zur ,Wissenssoziologie', in: Unter dem Banner des Marxismus 5 (1931) 83-102, hier: 101: "Die kleinbürgerlichen Utopien von gestern ferner werden, dem Schicksal des Kleinbürgertums im Geschichtsablauf gemäß, nie zur Wahrheit von morgen. ... Es handelt sich, im krassesten Gegensatz zu M.s Vorstellung, nicht um einen Wettlauf innerhalb der gleichen Erkenntnisebene, wo die Wahrheiten von gestern die Ideologien von heute, und wo die Utopien von heute die Wahrheiten von morgen wären. Es handelt sich vielmehr - und das ist eben die Kernansicht der Marxschen Lehre vom Wesen und von den Bedingungen des gesellschaftlichen Wissens - um strukturell verschiedenartige Erkenntnisprozesse, die auf verschiedenen gesellschaftlichen Klassenebenen vor sich gehen."

76 Siegfried Kracawer, Ideologie und Utopie, in: Literaturblatt. Beilage zur Frankfurter Zeitung (28. 4. 1929); Günther Stern, Über die sog. ,Seinsverbundenheit‘ des Bewußtseins. Anläßlich Karl Mannheims ,Ideologie und Utopie', in: Archiv für Sozialwissenschaft und Sozialpolitik 64 (1930) 492-509; Michael Freund, Karl Mannheim, Ideologie und Utopie, in: 
kleinere Modifikationen der sozialwissenschaftlichen Utopiekonzeption wurden von Paul Tillich, Karl Dunkmann, Siegfried Marck und Robert Heiß vorgeschlagen $^{77}$. Die Rezeption von „Ideologie und Utopie ${ }^{*}$ beschränkte sich jedoch nicht auf die politische Linke und die akademische Sozialwissenschaft, sondern auch auf der politischen Rechten wurde Mannheim positiv aufgenommen ${ }^{78}$.

Hier beschäftigte sich insbesondere der Leipziger Professor für Soziologie Hans Freyer (1887-1960) mit Fragen der Utopie bzw. des Utopischen in der Geschichte ${ }^{79}$. Freyers Denken speiste sich wesentlich aus der Gestalt der Kultur- und Sozialwissenschaften an der Universität Leipzig ${ }^{80}$, der Jugendbewegung und dem Spektrum des konservativen Utopismus ${ }^{81}$. Wie sein akademischer Lehrer Johann Plenge lehnte Freyer Positionen ab, die auf der Basis eines starken W/issenschaftlichkeitspostulats die Notwendigkeit konstruktiver Zukunftsentwürfe negier$\operatorname{ten}^{82}$. So erklärte Freyer in seinem Artikel „Das Problem der Utopie ${ }^{\star}$, der bereits 1914 geschrieben ${ }^{83}$, aber erst 1920 in der "Deutschen Rundschau“ veröffentlicht

Deutsche Literaturzeitung. Wochenschrift für Kritik der internationalen Wissenschaft, 3.F. 1 (1930) 2148-2156.

77 Paul Tillich, Ideologie und Utopie, in: Die Gesellschaft 6 (1929) 348-355 (wieder abgedruckt in: Meja, Stehr, Der Streit 451-458); Karl Dunkmann, Ideologie und Utopie. Nach dem gleichnamigen Buch von Karl Mannheim, in: Archiv für angewandte Sozialforschung 2 (1929) 71-83; Siegfried Marck, Zum Problem des "seinsverbundenen Denkens", in: Archiv für systematische Philosophie und Soziologie 33 (1929) 238-252 (wieder abgedruckt in: Meja, Stebr, Der Streit, 438-450); Robert Heiß, Mannheim, Karl: Ideologie und Utopie, in: Kölner Vierteljahrshefte für Soziologie 8 (1929) 240-243: Adolf Menzel kritisierte zwar Mannheims Utopiebegriff, weil er mit dem allgemeinen Sprachgebrauch nicht übereinstimme, aber das hinderte ihn nicht daran, der historischen Analyse des utopischen Bewußtseins zuzustimmen. Adolf Menzel, Ideologie und Utopie, in: Zeitschrift für Nationalökonomie 2 (1931) 408-417, bes. 414-417.

78 Z.B. Horst Grüneberg, Das Ende der Wissenschaft?, in: Die Tat 21 (1929) 597-608 (wieder abgedruckt in: Meja, Stebr, Der Streit 616-633). Auf die intensive Rezeption durch Hans Freyer wird im folgenden eingegangen.

79 Zu Freyers Biographie vgl. die hervorragende Studie von Jerry Z. Muller, The Other God That Failed. Hans Freyer and the Deradicalization of German Conservatism (Princeton, NJ 1987); im folgenden zitiert: Muller, The Other God; sowie die Arbeit von Elfriede Uner, Soziologie als "Geistige Bewegung“. Hans Freyers System der Soziologie und die „Leipziger Schule" (Weinheim 1992); im folgenden zitiert: Uner, Soziologie.

80 Dazu: Michael Geier, Der genius loci lipsiensis, in: Kultursoziologie 5 (1996) 109-131.

81 Zum Begriff des "konservativen Utopismus": Raimund von dem Bussche, Konservatismus in der Weimarer Republik. Die Politisierung des Unpolitischen (Heidelberg 1998) 383. 82 Johann Plenge, Marx und Hegel (Tübingen 1911) 169: "Marx aber strich das Sollen vollkommen aus seiner bewußten Gesellschaftslehre. ... so verfällt er doch in eine unterkapitalistische Beschränktheit, wenn er alle eine reale Zukunft vorbereitenden, mögliche Organisationsformen erfindenden Konstruktionen einer historisch denkenden Gesellschaftstechnik grundsätzlich verwirft. Weil phantastische Utopien da sind, kein konstruktives, organisatorisches Denken! Wie wenn einer aus Zorn über die phantasievollen Opfer des perperuum mobile das Erfinden von Maschinen verböte. $\mathrm{Zu}$ Plenge und seinem Verhältnis zu Freyer: $A x e l$ Schildt, Ein konservativer Prophet moderner nationaler Integration. Biographische Skizze des streitbaren Soziologen Johann Plenge (1874-1963), in: VfZ 35 (1987) 523-570, bes. 564.

${ }^{83}$ Zum Abfassungsdatum: Hans Freyer, Lebenslauf (1920), Universitätsarchiv Leipzig PA 474. 
wurde, die Utopie zu einem Willensrecht: „Wenn aber einer dies alles weiß und dennoch mit vorgreifender Phantasie vor sich und den Menschen, um deren Willen er wirbt, das Bild der Utopie aufrichtet, so tue er es. Denn dann ist sein Utopismus kein Denkfehler, sondern ein Willensrecht; durch keine Aufweisung eines inneren Widerspruchs widerlegbar, sondern ewig-gültig als eine schöpferische Form der praktischen Vernunft und als ein Weg jenes Strebens nach dem stärkeren, gesünderen, glücklicheren Menschentum, das in aller Naivität und Vertracktheit die tiefe Weisheit der Eschatologie bleibt und an dem wir ... alle miteinander mit dem Traum irgendeiner Hoffnung, mit einem klug verschwiegenen Plan oder einem kühnen Werke der Kultur gläubig teilnehmen, solange überhaupt die Kraft zum tätigen Leben in uns ist. ${ }^{84}$ Freyers Befürwortung der Utopie als gesellschaftsverändernde Kraft drückte sich auch in dem unbedingten Gestaltungs- und Handlungswillen seiner weniger wissenschaftlichen Schriften aus ${ }^{85}$. Damit bildete Freyer, wie bereits Alfred Doren 1923 erklärte, auf der politischen Rechten einen Gegenpol zu dem verbreiteten Geschichtspessimismus und Fatalismus Oswald Spenglers ${ }^{86}$ : „Dem skeptisch-rückschauenden Spenglermythus mit seiner Absage an jede geistig-künstlerische Entwicklungsmöglichkeit des alt und müde gewordenen Abendlandes tritt keck und siegesbewußt, kampfesfroh und herausfordernd Hans Freyer entgegen, der epimetheischen Weltanschauung eines ungeheure Weiten umspannenden Denkers die promethische Hoffnung auf geistigen Segen, geboren aus der Kraft eines freudigen, reinen, unbeschwerten Willens, entgegenstellend. ${ }^{\alpha 7}$

Auch in Hans Freyers Konzeption einer "Soziologie als Wirklichkeitswissenschaft" spielte der Utopiebegriff, den er in expliziter Anlehnung an die Definition von Karl Mannheim entwickelte, eine zentrale Rolle $e^{88}$. Wenn der Soziologe die Wirklichkeit erkennen wolle, so Freyer, müsse er auch die Tendenzen erfassen, die die Gegenwart transzendieren und verändern könnten. Weil jedoch erst die Zukunft erweisen könne, welches die wirkliche seinsverändernde Kraft, d.h. die richtige Utopie sei, könne dies nicht wissenschaftlich bestimmt werden. Vielmehr müsse eine Utopie bzw. eine gesellschaftliche Willenstendenz vom Soziologen als

84 Hans Freyer, Das Problem der Utopie, in: Deutsche Rundschau 183 (1920) 321-345, hier: 345.

85 Vgl. z. B. Hans Freyer, Antäus. Grundlegung einer Ethik des bewussten Lebens (Jena 1918) 4; Hans Freyer, Prometheus. Ideen zu einer Philosophie der Kultur (Jena 1923) 111, 113: „die Zukunft aber: das ist das Nichts, aus dem unablässig die Wunder des Gegenwärtigen quellen (... und ihr) Sinn ist nicht Dauer sondern Bruch, der Sinn der Gegenwart nicht Erfüllung sondern Bereitschaft, der Sinn des Lebens nicht Glauben sondern Schaffen."

$86 \mathrm{Vgl}$. Spenglers Antiutopismus in: Oswald Spengler, Der Mensch und die Technik. Beitrag zu einer Philosophie des Lebens (München 1931) 6; Oswald Spengler, Jahre der Entscheidung. Erster Teil. Deutschland und die weltgeschichtliche Entwicklung (München 1933) 4; und Freyers Rezension von „Der Untergang des Abendlandes“: Hans Freyer, Der Untergang des Abendlandes, in: Die Tat 11 (1919) 304-308, bes. 308.

${ }^{87}$ Alfred Doren, Von Spengler zu Freyer, in: Neue Zürcher Zeitung (17. 8. 1923).

${ }^{88}$ Hans Freyer, Soziologie als Wirklichkeitswissenschaft. Logische Grundlagen des Systems der Soziologie (Leipzig, Berlin 1930) 298; im folgenden zitiert: Freyer, Soziologie. 
wahr und gültig gesetzt werden, um die Wirklichkeit zu begreifen ${ }^{89}$. Da die soziologische Analyse selbst wieder auf die Gesellschaft wirken sollte, konzipierte Freyer seine Soziologie also in zweifachem Sinn als Transformationswissenschaft: als Wissenschaft von den gesellschaftlichen Veränderungstendenzen und als Beitrag zur Veränderung der Gesellschaft.

Nach der Zahl der Rezensionen zu urteilen, rief Freyers „Soziologie als Wirklichkeitswissenschaft" bis 1933 ein zumindest ebenso großes Echo hervor wie Mannheims „Ideologie und Utopie ${ }^{40}$. Dabei kreisten die Diskussionen insbesondere um Freyers willensmäßige Fundierung soziologischer Erkenntnis. Während liberale Sozialwissenschaftler diese Idee ablehnten ${ }^{91}$, stimmten Vertreter der politischen Rechten oder Linken Freyer emphatisch zu'92. Dabei stellten beide Seiten

89 Ebd. 298-307, 304: „Jeder Strukturbegriff der gegenwärtigen Gesellschaftsordnung setzt voraus, daß ein bestimmter Wille, diese Sozialstruktur künftig umzubilden, ihre (sic) diese uder jene Entwicklungsrichtung zu geben, als geschichtlich gültig gesetzt oder anerkannt werde."

90 Es erschienen insgesamt mindestens 33 Rezensionen, und zwar 1931 in: Archiv des öffentlichen Rechts, Archiv für angewandte Soziologie, Archiv für Sozialwissenschaft und Sozialpolitik, Zeitschrift für deutsche Philosophie, Das Literarische Centralblatt für Deutschland, Heimatbildung, Jahrbuch für Gesetzgebung, Verwaltung und Volkswirtschaft im Deutschen Reich, Kantstudien, Kindergarten, Vergangenheit und Gegenwart, Kölner Vierteljahrshefte für Soziologie, Die Volksschule, Zeitschrift für Handelsschulpädagogik, Zeitschrift für Nationalökonomie, Zeitschrift für angewandte Psychologie, Kölnische Zeitung, Leipziger Neueste Nachrichten, Neue Zürcher Zeitung. Im Jahr 1932 in: Archiv für Rechts- und Wirtschaftsphilosophie, Deutsche Blätter für Philosophie, Bücherwarte, Evangelisch-Sozial, Die Gesellschaft, Philosophisches Jahrbuch der Görres-Gesellschaft, Monatsschrift für Kriminalpsychologie, Zeitschrift für schweizerische Geschichte, Zeitschrift für Sozialforschung, Zeitschrift für die gesamte Staatswissenschaft, Frankfurter Zeitung. Und 1933 in: Philosophische Hefte, Historisches Jahrbuch der Görres-Gesellschaft, Jahrbücher für Nationalökonomie und Statistik, Vierteljahrsschrift für Sozial- und Wirtschaftgeschichte.

91 Andreas Walther, Das Problem einer deutschen' Soziologie, in: Kölner Vierteljahrshefte für Soziologie 9 (1931) 513-530, 518; Gerhard Lehmann, Freyers "Soziologie als Wirklichkeitswissenschaft “, in: Archiv für angewandte Soziologie 3 (1931) 205-211, 208; Josef Pieper, "Wirklichkeitswissenschaftliche“ Soziologie. Kritische Randbemerkungen zu Hans Freyers "Soziologie als Wirklichkeitswissenschaft, in: Archiv für Sozialwissenschaft und Sozialpolitik 66 (1931) 394-407, 404. Karl Mannheim lehnte Freyers Position nach anfänglicher Zustimmung (so Muller, The Other God 182) 1932 auf der Frankfurter Dozententagung mit deutlichen Worten ab: „Mündet diese Theorie gar in den überpointierten Satz: Wahres Wollen fundiert wahre Erkenntnis', so öffnen wir in der Theorie jeder Beliebigkeit Tor und Tür. Denn wer wird wohl in der Arena der Gedanken nicht in der Überzeugung oder in der Maske des, wahren Wollens' auftreten, und wer wird sich in dieser Situation nicht freuen, daß er sich von nun an nicht mehr nur sachhaltig ausweisen muB, sondern daß es erlaubt sein wird, sich im Erkennen auf Eingebung und ,wahre Gesinnung' zu berufen." Zitat bei Karl Mannheim, Die Gegenwartsaufgaben der Soziologie (Tübingen 1932) 40.

92 Alfred Kleinberg, Soziologie der goldenen Mitte. Zu Hans Freyers, Soziologie als Wirklichkeitswissenschaft', in: Die Gesellschaft 9 (1932) 68-74, 73 f.; Gerbard Ledig, Hans Freyers Soziologie und der Sozialismus, in: Neue Blätter für den Sozialismus 2 (1931) 291-294, bes. 291; Herbert Marcuse, Zur Auseinandersetzung mit Hans Freyers „Soziologie als Wirklichkeitswissenschaft", in: Philosophische Hefte (1931) 83-91. Die positive Rezeption auf der Rechten äußerte sich durch die Neubesetzung des Utopiebegriffs wie z. B. in der Zeit- 
das grundsätzliche Verständnis der Utopie als individuelle oder kollektive Bewußtseinsform, die gesellschaftliche Veränderung herbeiführt - genauso wie in der Diskussion um Mannheims „Ideologie und Utopie“ - nicht in Frage. Daher kann die Etablierung der sozialpsychologischen Diskursformation mit der breiten inner- und außerwissenschaftlichen Rezeption von Hans Freyer und Karl Mannheim als abgeschlossen betrachtet werden.

\section{Vom hellblauen Seidenhemd zum Gestaltbarkeitsbewußtsein - Kontextualisierungen}

\section{Personelle und intellektuelle Verbindungen}

Neben dem Rückbezug der meisten zentralen Figuren des Utopiediskurses der 20er Jahre auf Voigts "Die Sozialen Utopien" und Landauers "Die Revolution" lag ein Grund für die Etablierung einer einheitlichen Diskursformation auf persönlich-biographischer Ebene: Die verschiedenen Diskursspektren waren nicht nur intern ${ }^{93}$, sondern auch untereinander eng vernetzt. Ernst Bloch und Karl Mannheim waren trotz Mannheims negativer Rezension von Blochs „Geist der

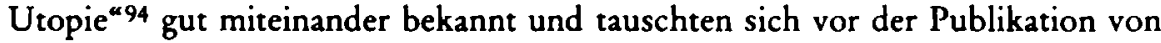
"Ideologie und Utopie" auch über Fragen der Utopie aus. So ließ Bloch 1928 Siegfried Kracauer von Mannheim grüßen und berichtete ihm, Mannheim verfasse einen Text über ${ }_{n}$ das utopische Bewußtsein ${ }^{495}$. Eine enge intellektuelle Verbindung zwischen Bloch und Mannheim bestand zudem dadurch, daß beide in ihrer Jugend längere Zeit mit Georg Lukács befreundet waren. Allerdings nahm Lukács für die Utopietheoriebildung der beiden eine unterschiedliche Rolle ein: Während er für Bloch zunächst sozusagen ein Bruder im utopischen Geiste gewesen war ${ }^{96}$ und dann zum Referenzpunkt für seine Absetzbewegung vom orthodoxen Marxismus wurde97, stellte Lukács - wie auch Gustav Landauer - mit

schrift „Die Tat ${ }^{\alpha}$. Vgl. Hans Zehrer, Um die politische Jugend. Utopie und Realität, in: Die Tat 20 (1928) 227-229; im folgenden zitiert: Zebrer, Jugend. Als kritische Stimme, ohne jedoch den sozialwissenschaftlichen Utopiebegriff letztlich in Frage zu stellen: Georg Quabbe, Das letzte Reich. Wandel und Wesen der Utopie (Leipzig 1933) bes. 5f. und 117-121.

${ }^{93}$ Für das Spektrum der akademischen Sozialwissenschaften ist dies selbstverständlich. Siehe: Dirk Käsler, Die frühe deutsche Soziologie und ihre Entstehungs-Milieus. Eine wissenschaftssoziologische Untersuchung (Opladen 1984). Zum linken Diskursspektrum siehe Anm. 53 und zum rechten Diskursspektrum um Freyer: Muller, The Other God 17, 150.

94 Karl Mannheim, Ernst Bloch: Geist der Utopie, in: Athenaeum (1919) 207-212.

95 Bloch, Briefe I 303. Zur Bekanntschaft von Bloch und Mannheim siehe auch: Bloch, Briefe II 785 und den Brief Benjamins an Gershom Scholem vom 30. 12. 1922, in: Walter Benjamin, Gesammelte Briefe, Bd. 2 (Frankfurt a.M. 1996) 299. Woldring berichtet, Mannheim habe mit Bloch und Buber zusammen in Heidelberg regelmäßig an einer Diskussionsrunde teilgenommen. Woldring, Karl Mannheim 21.

\% Vgl. die Selbstzeugnisse wie in Anm. 53 nachgewiesen.

97 Siehe: Ernst Bloch, Akrualität und Utopie. Zu Lukács Geschichte und Klassenbewusstsein, in: Der neue Merkur 7.7 (1923/24) 457-477. 
seinem Engagement in der Räterepublik für Mannheim eher ein Anschauungsobjekt für das Studium der W/rkung des utopischen Bewußtseins in der Geschichte dar ${ }^{98}$.

Mit seinem Ruf an die Frankfurter Universität, wo er 1930 die Nachfolge von Franz Oppenheimer (1864-1943) als Professor für Soziologie antrat ${ }^{99}$, intensivierten sich Mannheims Beziehungen zum Spektrum linker und marxistischer Intellektueller zwangsläufig, da die Universitätsleitung sein Büro und das neugegründete soziologische Institut im Gebäude des Instituts für Sozialforschung einrichtete ${ }^{100}$. Hier stellte Mannheim eine Herausforderung für die intellektuelle Hegemonie der frühen Frankfurter Schule dar ${ }^{101}$, weil er eine große Anziehungskraft auf Studierende und junge Wissenschaftler ausübte, die nicht zuletzt in seinem Vortragsstil und seinen hellblauen Seidenhemden begründet lag ${ }^{102}$. In der Forschung werden die etwas unterkühlten Beziehungen zwischen Mannheim und der Frankfurter Schule gemeinhin auf die theoretischen Differenzen zwischen Wissenssoziologie und kritischer Theorie zurückgeführt ${ }^{103}$. Vor dem Hintergrund der intellektuellen Konkurrenzsituation an der Frankfurter Universität ist es jedoch plausibler, in genau dieser eine wesentliche Ursache sowohl für die theoreti-

98 Mannheim, Ideologie 195f., 217. Zur Haltung von Lukács während der ungarischen Räterepublik: David Kettler, Culture and Revolution. Lukács in the Hungarian Revolutions of 1918/1919, in: Telos 10 (1971) 35-92, 73 und passim; im folgenden zitiert: Kettler, Culture. Darüber hinaus wurde Mannheim natürlich auch von Lukács' theoretischen Positionen beeinflußt. Zur Wirkung von "Geschichte und Klassenbewußtsein" auf Mannheims Denken: Congdon, Exile 290; Loader, Karl Mannheim 77f.; Kettler, Meja, Karl Mannheim 36-38, 46 und 85. Noch im Winter 1928/29 hielt Mannheim mit Alfred Weber ein Seminar über das Buch ab. So: Woldring, Karl Mannheim 29.

${ }_{99} \mathrm{Zu}$ den genauen Berufungsmodalitäten siehe: Woldring, Karl Mannheim 29-32; Paul Kluke, Die Stifungsuniversität Frankfurt am Main 1914-1932 (Frankfurt a.M. 1972) 545; und Notker Hammerstein, Die Johann Wolfgang Goethe-Universität Frankfurt am Main. Von der Stiftungsuniversität zur staatlichen Hochschule, Bd. 1: 1914-1950 (Neuwied 1989) 128-130; im folgenden zitiert: Hammerstein, Die Johann Wolfgang Goethe-Universität.

$100 \mathrm{Vgl}$.: Hammerstein, Die Johann Wolfgang Goethe-Universität 300.

101 Zur intellektuellen Hegemonie der Frankfurter Schule: Wolfgang Schivelbusch, Intellektuellendämmerung. Zur Lage der Frankfurter Intelligenz in den zwanziger Jahren (Frankfurt a. M. 1982) 14-28; und Hammerstein: Die Johann Wolfgang Goethe-Universität 62.

102 Vgl.: Kurt $H$. Wolff, Wie ich zur Soziologie kam und wo ich bin (Interview mit Nico Stehr), in: Soziologie in Deutschland und Österreich 1918-1945, hrsg. v. M. Rainer Lepsius (Opladen 1981) 324-346, 324: „Nach einem Jahr Studium etwa ... sagte mir jemand, da wäre ein fabulöser Mann aus Heidelberg gekommen. Der war le dernier cri, man mußte ihn einfach hören, und ich bin hin. Sein Name war Karl Mannheim, und ich war sofort fasziniert von ihm - hauptsächlich, erinnere ich mich, aus zwei Gründen: Erstens trug er Seidenhemden, und zwar hellblaue, zweitens hatte er einen ungarischen Akzent." Siehe auch: Ulf Matthiesen, Kontrastierungen/Kooperationen. Karl Mannheim in Frankfurt (1930-1933), in: Die (mindestens) zwei Sozialwissenschaften in Frankfurt und ihre Geschichte, hrsg. v. Heinz Steinert (Frankfurt a. M. 1989) 72-87, hier: 72; und Kettler, Meja, Karl Mannheim 121.

$103 \mathrm{Vgl}$. als Beispiel Leon Bailey, Critical Theory and Sociology of Knowledge. A Comparative Study in the Theory of Ideology (New York 1994) 63: „Undoubtedly this distance was largely due to the serious substantive disagreements that served to divide Mannheim from the Frankfurt School.“ 
schen als auch für die persönlichen Differenzen zu suchen ${ }^{104}$. Zur direkten intellektuellen Konfrontation - auch über Fragen der Utopie - kam es zum Beispiel in dem regelmäßig von Paul Tillich organisierten Gesprächskreis, dem sogenannten "Kränzchen" 105 .

Tillich führte nicht nur Karl Mannheim und die Frankfurter Schule zusammen, sondern es ergab sich über ihn auch eine Verbindung zu Hans Freyer. Tillich und Freyer waren beide Mentoren und geistige Bezugspunkte des jugendbewegten Leuchtenburgkreises, der 1924 unter der Leitung von Fritz Borinski bei Jena gegründet wurde ${ }^{106}$. Ein direkter Gedankenaustausch zwischen Tillich und Freyer könnte auch an der Leipziger Universität stattgefunden haben, da Tillich dort von 1927 bis 1929 als Honorarprofessor unterrichtete und wie Hans Freyer an dem von Hermann Heller eingerichteten Seminar für Volksbildungswesen Kurse abhielt ${ }^{107}$. An der Universität stand Freyer auch in Kontakt zu Alfred Doren, mit dem zusammen er die Dissertation Roland Haases zum Thema Chiliasmus betreute ${ }^{108}$. Weitere Verbindungen zwischen den Leipziger und den Frankfurter Utopietheoretikern ergaben sich zudem, weil Karl Mannheims Cousin Ernst Manheim Assistent bei Hans Freyer war und sich einige Schüler Freyers später im Umfeld der Frankfurter Schule wiederfanden ${ }^{109}$.

Darüber hinaus ist den Lebensläufen von Hans Freyer, Karl Mannheim und Ernst Bloch gemeinsam, daß sie alle zumindest ein Semester bei Georg Simmel (1858-1918) in Berlin studierten ${ }^{110}$. Wenngleich ein Studium bei Simmel ein Muß

104 Eine weitergehende Analyse im Stile Pierre Bourdieus müßte hier versuchen, die theoretischen Positionen genauer zu den jeweiligen Stellungen im sozialen Raum der Universität in Beziehung zu setzen. Vgl.: Pierre Bourdieu, Homo academicus (Frankfurt a. M. 1988) bes. 17. 105 Hier traf sich der Freundeskreis von Paul Tillich, vornehmlich bestehend aus religiösen Sozialisten, der frühen Frankfurter Schule, aber auch anderen Frankfurter Wissenschaftlern. Vgl. Martin Jay, The Dialectical Imagination. A History of the Frankfurt School and the Institute of Social Research 1923-1950 (Berkeley, Los Angeles, London 1996, 1. Aufl. 1971) 24. Vgl. zum Thema Utopie die Mitschrift der Diskussion vom 27. Juni 1932 zum Thema "Die säkulare Zivilisation und die Missionsaufgabe des Christentums", in: Paul Tillich, Briefwechsel und Streitschriften (Frankfurt a.M. 1983) 314-369.

106 Wemer Kindt, Hans Raupach (Hrsg.), Die deutsche Jugendbewegung 1920 bis 1933. Die bündische Zeit. Quellenschriften (Düsseldorf, Köln 1974) 1045-1049.

107 Wilhelm u. Marion Pauck, Paul Tillich. Sein Leben und Denken, Bd. 1: Leben (Stuttgart, Frankfurt a. M. 1978) 117; und Uner, Soziologie 14f. Zu Freyers Engagement in der Erwachsenenbildung siehe auch: Helene Kleine, Soziologie und die Bildung des Volkes. Hans Freyers und Leopold v. Wieses Position in der Soziologie und der freien Erwachsenenbildung während der Weimarer Republik (Opladen 1989) bes. 94-103.

108 Roland Haase, Das Problem des Chiliasmus und der Dreißigjährige Krieg (Diss. Leipzig 1933). Ihre Beziehung war zumindest so gut, daß Hans Freyer als Dekan zugunsten von Alfred Doren intervenierte, als dieser 1933 aufgrund des Gesetzes zur „Wiederherstellung des Berufsbeamtentums" seine Lehrtätigkeit aufgeben mußte. Gerald Diesener, Jaroslav Kudma, Alfred Doren (1869-1934), in: Storia della Storiografia 23 (1993) 25-45, hier: 43. 109 Siehe: Muller, The Other God 89; und Uner, Soziologie 3.

110 Bloch und Freyer hatten währenddessen intensiveren Kontakt zu Simmel. Muller, The Other God 45; Münster, Tagträume 33-36; sowie Münster, Utopie 54; zu Mannheim: Kettler, Meja, Karl Mannheim 3; und Woldring, Karl Mannheim 6. 
für aufstrebende Soziologen im späten Kaiserreich gewesen ist ${ }^{111}$, liegt es dennoch nahe, hier einen Katalysator für ihre Behandlung der Utopien bzw. des Utopischen zu suchen. Denn Simmel begriff die Setzung von Endzielen als eine wesentliche Bedingung der menschlichen Existenz und diagnostizierte in ihrem Fehlen durch den Verlust der Verbindlichkeit von Religion ein Grundproblem der Moderne $^{112}$. Vor diesem Hintergrund kann man Mannheims, Freyers und Blochs theoretische Beschäftigung mit dem Phänomen der Utopie als wissenschaftliche Untersuchung des modernen Versuchs verstehen, den Religionsverlust durch Utopien zu kompensieren.

Die so aufgezeigten biographischen Vernetzungen beeinflußten die Etablierung des sozialpsychologischen Utopieverständnisses in zweifacher Weise: Zum einen führten sie dazu, daß sich der Diskurs nicht nur in Aufsätzen und Büchern manifestierte, sondern die Utopie bzw. das Utopische auch Gegenstand von Gesprächen und Diskussionen war. Zum anderen erhöhte die wechselseitige persönliche Bekanntschaft und Vernetzung zwischen den verschiedenen Diskursspektren die Rezeptivität für die Meinungen der jeweils anderen. Dies führte $z$ war nicht zur kritiklosen Annahme der Thesen des Gegenübers, wie die Rezeption von Mannheims "Ideologie und Utopie" durch die Frankfurter Schule zeigt, aber es trug zur Vereinheitlichung der Diskursformation bei.

\section{Krisenwahrnehmung und Utopie}

Ein weiterer wichtiger Kontext für die Entstehung des sozialwissenschaftlichen Utopiediskurses war der Zusammenhang von Krise und Utopie: Die meisten der behandelten Autoren nahmen die Gegenwart als Krise wahr und reagierten darauf mit der Formulierung einer Utopie. Was als Krise angesehen und was dieser als Utopie entgegenstellt wurde, variierte dabei in Abhängigkeit vom politischen und intellektuellen Hintergrund der Autoren.

Der Anarchist Gustav Landauer diagnostizierte genauso wie Ernst Bloch nicht nur eine Krise des Kapitalismus und des Staates, sondern auch der sozialistischen Bewegung, deren Ursache er in der Utopievergessenheit bzw. der Vernachlässigung des geistigen Elements im Marxismus und den sozialistischen Parteien sah $^{113}$. Als Ausweg aus der Verfestigung der kapitalistischen Staats- und Wirtschaftsordnung formulierte Landauer die Utopie eines dezentralen Zusammen-

111 David Frisby, Georg Simmel (Chichester, London, New York 1984) 32 ff.

112 Vgl. Georg Simmel, Schopenhauer und Nietzsche (1907), in: Gesamtausgabe Bd. 10, hrsg. v. Michael Behr, Volkhard Knecht, Gert Schmidt (Frankfurt a. M. 1995) 169 und 176-178. Zu dieser Position Simmels siehe: David Frisby, Fragmente der Moderne. Georg Simmel - Siegfried Kracauer - Walter Benjamin (Oxford 1986) 49f. und Klaus Licbiblau, Georg Simmel (Frankfurt a. M., New York 1997) 115, 118.

113 Gustav Landauer, Aufruf zum Sozialismus. Ein Vortrag (Berlin 1911) 2, 6, 29-66 und 116 f.; im folgenden zitiert: Landauer, Aufruf. Daß Landauer die Gegenwart als Krise wahrnahm, macht auch sein eigenes Engagement in der Räterepublik deutlich, das nach seiner Theorie nur in Krisenzeiten sinnvoll war, weil nur dann die Utopie Massenzuspruch erlangen konnte. 
schlusses freier Gemeinden und Siedlungsgenossenschaften, in denen durch die Neuaufteilung des Bodens und die Aufhebung des kapitalistischen Tauschprinzips ein neuer Geist herrschen solle114. Ernst Blochs Utopie, die die durch die gescheiterte Revolution intensivierte Krise überwinden sollte ${ }^{115}$, war die Vorstellung einer sozialistischen Brüdergemeinde, die chiliastische Elemente mit Vorstellungen einer kommunistischen Wirtschaftsordnung verband ${ }^{116}$.

Für Karl Mannheim bestand die Krise in der Fragmentierung und Segmentierung der politischen und wissenschaftlichen Kultur im Deutschland der 20er Jahre sowie in der im Sinne von Ernst Troeltsch verstandenen „Krise des Historismus " ${ }^{117}$. Der Wunsch, diese Krise zu überwinden, war eine wesentliche Motivation für die Entwicklung seiner $W_{1 s s e n s s o z i o l o g i e}{ }^{118}$, an deren Ende die Utopie einer Überwindung der politischen und kulturellen Gegensätze durch die Syntheseleistung der "freischwebenden Intelligenz" ${ }^{\text {stand }}{ }^{119}$.

Auf der politischen Rechten definierte Hans Freyer den Verlust der alten Ordnung durch die tiefe Klassenspaltung der Industriegesellschaft als zentrales Krisenphänomen, das durch den Verlust des revolutionären Potentials der Arbeiterklasse noch verschärft werde ${ }^{120}$. Die Fragmentierung der industriellen Klassengesellschaft sollte überwunden werden in der Utopie einer neuen Gemeinschaft in einem neuen Staat, die durch eine Revolution des Volkes, eine „Revolution von rechts", zu realisieren sei ${ }^{121}$.

114 Landauer, Aufruf 139-149; Landauer, Revolution 117. Dazu: Norbert Altenhofer, Tradition als Revolution: Gustav Landauers, geworden-werdendes' Judentum, in: Jews and Germans from 1860 to 1933, hrsg. v. David Bronsen (Heidelberg 1979) 173-208, bes. 187-191; vgl. Braun, Die Siedlung.

$115 \mathrm{Vgl}$. Blochs Selbstzeugnis in Münster, Tagträume 40.

116 Bloch, Geist (1918) 410, 444 f.; Bloch, Geist (1923) 328 f.

117 Vgl. z.B.: Karl Mannheim, Heidelberger Briefe (1921 f.), in: Karádi, Vézer, Sonntagskreis 72-91, 80.

118 Vgl.: Mannbeim, Ideologie 51: „Weil dieses Buch sich einer Krisensituation des Denkens bewußt ist, an den Aussichten der Lösbarkeit aber nicht zweifelt ...". Auf den Aspekt der Krise und ihrer Lösbarkeit beziehen sich insbesondere die folgenden Rezensionen: Robert Heiß, Mannheim, Karl: Ideologie und Utopie, in: Kölner Vierteljahrshefte für Soziologie 8 (1929) 240-243; Edrard Spranger, Ideologie und Wissenschaft, in: Forschungen und Fortschritte 6 (1930) $131 \mathrm{f}$. (wieder abgedruckt in: Meja, Stehr, Der Streit 634-636) und Michael Freund, Karl Mannheim, Ideologie und Utopie, in: Deutsche Literaturzeitung, 3. F. 1 (1930) 2148-2156.

119 Mannbeim, Ideologie 128-143. Vgl. dazu: Frisby, Mind 24, 159-162. So auch David Kettler, Volker Meja, Nico Stehr, Politik als Wissenschaft. Über Theorie und Praxis bei Karl Mannheim, in: Angewandte Sozialforschung 11 (1983) 403-417, 412: „Mannheim verstand sein eigenes Werk immer als eine Arbeit des Uberganges, die durch eine Krise in der liberalen Tradition und Ordnung notwendig wurde, und er machte es zu seinem erklärten Ziel, eine Synthese zu entwickeln, welche die partielle Legitimität jeder der unerbittlich kämpfenden Parteien anerkennen und begreifen würde, um die theoretischen und politischen Bereiche wieder zu versöhnen."

120 Hans Freyer, Revolution von rechts (Jena 1931) 34, im folgenden zitiert: Freyer, Revolution.

121 Freyer, Revolution 43 f., 49-55. Hans Freyer, Der Staat (Leipzig 1925) bes. 130-216. 
Grundsätzlich kann man feststellen, daß der eigene Utopismus bzw. der von anderen geäußerte Utopismusvorwurf häufig weitergehende theoretische Reflexionen über den Utopiebegriff auslöste, die zu einer ${ }_{n}$ progressiven Historisierung der Utopiekritik“ (Lucian Hölscher) und einer positiven Bewertung der Utopien führten ${ }^{122}$. So entgegnete Franz Oppenheimer 1925 auf den Vorwurf, seine ländlichen Siedlungsgenossenschaften im Stile Theodor Hertzkas seien eine Utopie ${ }^{123}$ : "Alle Wirklichkeit ist die Utopie von gestern ...", und „... alle Utopie ist die Wirklichkeit von morgen. ${ }^{~} 124$ In gleicher Weise sah sich Otto Neurath wegen seiner Pläne einer "Vollsozialisierung" der Wirtschaft mit dem Utopismusvorwurf konfrontiert ${ }^{125}$ und reagierte mit der These, die Konstruktion von Utopien sei notwendig zur rationalen Gestaltung der zukünftigen Gesellschaft ${ }^{126}$. Auch der spätere Redakteur der Zeitschrift „Die Tat", Hans Zehrer (1899-1966), verteidigte sich gegen den Vorwurf, sein Vorschlag zu einem Zusammenschluß der Jugendverbände sei eine Utopie, mit dem Verweis auf die Funktion der Utopie in historischen Veränderungsprozessen: „Freilich, was ist Utopie? Es ist eine Zielsetzung mit den im Augenblick verfügbaren Mitteln. Je unzureichender die Mittel sind, desto utopischer erscheint das Ziel. Aber die Mittel sind dauernd im Fluß, sie wandeln sich und werden bedingt durch die Situation. ${ }^{{ }^{127}}$

Folglich war die Herausbildung des sozialpsychologischen Utopiediskurses auch eine Reaktion auf die Formulierung der Utopien, mit denen die Diskursteilnehmer die Krise der Zeit überwinden wollten: Indem in der sozialpsychologischen Diskursformation der mentalisierten Utopie eine zentrale Rolle in historischen Transformationsprozessen zugemessen wurde, rechtfertigte der Diskurs aufgrund der weit verbreiteten Wahrnehmung von Krisen und Veränderungsbedarf - den Utopismus der Diskursteilnehmer.

\section{Sozialwissenschaftler und politische Intellektuelle}

Neben dem Zusammenhang von Krisenwahrnehmung, Utopie und Utopietheorie auf der individuellen Ebene der Diskursteilnehmer bildete das Verhältnis von Utopie und Sozialwissenschaften sowie das Selbstverständnis der Diskursteilnehmer als politische Intellektuelle einen weiteren wichtigen Kontext für die Herausbildung der sozialpsychologischen Formation des Utopiediskurses. In der Forschung wird spätestens seit Karl Mannheim die Entstehung der Soziologie als eine

122 Zum Phänomen der „progressiven Historisierung “ im 19. Jahrhundert: Hölscher, Utopie 782.

${ }^{123}$ Als kurze Zusammenfassung seiner utopischen Vorstellungen: Franz Oppenbeimer, Reale Utopie, in: Die Wirtschaftswende. Zeitschrift für deutsche Wirtschaftserneuerung (1932) 821-828.

124 Franz Oppenheimer, System der Soziologie, Bd. 2: Der Staat (Stuttgart 21964 ) 730.

125 Neuraths Pläne: Otto Neurath, Durch die Kriegswirtschaft zur Naturalwirtschaft (München 1919). Die Entwicklung seiner utopietheoretischen Überlegungen in Entgegnung auf den Utopismusvorwurf: ebd. 228.

126 S.o. Anm. 51.

127 Zebrer, Jugend 227. 
Reaktion auf die verschiedenen Krisenerscheinungen und Krisenwahrnehmungen verstanden, die am Ende des 19. Jahrhunderts einsetzten und sich im Ersten Weltkrieg verschärften ${ }^{128}$. Desgleichen stellte die Inflation utopischen Denkens sowie der utopischen Literatur seit den 1890er Jahren, die eine weitere Intensivierung während des Ersten Weltkrieges und der revolutionären Prozesse in Rußland und anderen europäischen Staaten erfuhr, eine Suche nach Auswegen aus der als krisenhaft empfundenen Moderne dar ${ }^{129}$. Dieser gleiche Ursprung utopischen und sozialwissenschaftlichen Denkens in den Krisenerscheinungen der Zeit bzw. der Krisenwahrnehmung der Zeitgenossen spiegelte sich in der Ausrichtung vieler soziologischer Theorien auf die Utopie einer neuen Gemeinschaft ${ }^{130}$.

Daher war die Thematisierung der Utopie durch die Sozial wissenschaftler nicht nur eine Behandlung der Suche nach utopischen Wegen aus der Krise der Moderne, sondern gleichzeitig auch eine Selbstthematisierung der Sozialwissenschaften und ihrer Rolle in den als notwendig erachteten Veränderungsprozessen. Indem sie die Utopie im Rahmen der sozialpsychologischen Diskursformation als Bewußtseinsform verstanden und ihr eine zentrale Bedeutung in historischen Transformationsprozessen zumaßen, wiesen Sozialwissenschaftler wie Plenge, Mannheim, Freyer oder Neurath ihrer wissenschaftlichen Arbeit bzw. sich selbst - sozusagen als Experten für das Utopische - eine wesentliche Rolle in diesen Prozessen zu. Sie verstanden sich nicht nur als Wissenschaftler, sondern auch als

128 Karl Mannheim, German Sociology (1918-1933), in: Politica 1 (1934) 12-33, bes. 13. Vgl. z. B.: Klaus Lichtblau, Kulturkrise und Soziologie um die Jahrhundertwende. Zur Genealogie der Kultursoziologie in Deutschland (Frankfurt a.M. 1996); Karl Acham, Die "kulturelle" Krise der Gesellschaft und die Genese der Sozialwissenschaften, in: Vom Weltbildwandel zur Weltanschauungsanalyse. Krisenwahrnehmung und Krisenbewältigung um 1900 (Berlin 1996) 39-67; Erhard Stölting, Akademische Soziologie in der Weimarer Republik (Berlin 1986) 92; Jürgen Habermas, Soziologie in der Weimarer Republik, in: Wissenschaftsgeschichte seit 1900. 75 Jahre Universität Frankfurt, hrsg. v. Helmut Coing u.a. (Frankfurt a. M. 1992) 29-53, bes. $29 \mathrm{f}$.

$129 \mathrm{Vgl}$. zur allgemeinen Intensivierung ab 1890: Lucian Hölscher, Die Entdeckung der Zukunft (Frankfurt 2.M. 1999) 129-140; im folgenden zitiert: Hölscher, Entdeckung; Jost Hermand, Der alte Traum vom neuen Reich. Völkische Utopien und Nationalsozialismus, (Frankfurt a.M. 1988) 14; zur verstärkenden Wirkung von Weltkrieg und Revolution: Peter S. Fisher, Fantasy and Politics. Visions of the Future in the Weimar Republic (Madison 1991) 21-23. Diese wurde bereits von den Zeitgenossen wahrgenommen. Vgl. z. B. die Diskussionen um Otto Neurath und Wofgang Schumann sowie Ernst Bloch. Als charakteristische ÄuBerung für die Utopietheorie der unmittelbaren Nachkriegszeit: Semi Meyer, Utopie und Entwicklung, in: Nord und Süd 44 (1919) 141-147, 141: „Die Utopie erlebte durch den Krieg eine neue Blütezeit, eine Auferstehung ... Zum Aufkommen apokalyptischer Deutungsmuster im Ersten Weltkrieg siehe: Jay Winter, Sites of Memory. Sites of Mourning. The Great War in European Cultural History (Cambridge 1995) bes. 145; Anson Rabinbach, In the Shadow of Catastrophe. German Intellectuals between Apocalypse and Enlightenment (Berkeley, Los Angeles, London 1997) bes. 6, 8.

130 Diese wurde für Tönnies, Troeltsch, Weber, Simmel und Lukács nachgewiesen von: Harry Liebersobn, Fate and Utopia in German Sociology, 1870-1923 (Cambridge, Mass. 1988). In die gleiche Richtung argumentiert auf breiterer Quellenbasis: Paul Nolte, Die Ordnung der deutschen Gesellschaft. Selbstentwurf und Selbstbeschreibung im 20. Jahrhundert (München 2000) 28 und 61-207. 
politische Intellektuelle und sahen ihre wissenschaftliche Arbeit als Weg aus der Krise der Zeit ${ }^{131}$.

In diesem Sinne erklärte Gustav Landauer, seine Sozialpsychologie vergegenwärtige die utopischen Potentiale der Vergangenheit und sei somit ${ }_{n}$ selbst nichts anderes als die Revolution" ${ }^{132}$. Die Realisierung von Otto Neuraths Forderung einer ,wissenschaftlichen Utopistik " und einer an ihr ausgerichteten Politik hätte ihm und seinen Kollegen als Lehrmeistern der Utopistik die Gestaltung der zukünftigen Gesellschaft überlassen ${ }^{133}$. Auch Freyers „Soziologie als Wirklichkeitswissenschaft" wurde von dem "Traum" getragen, "daß die Soziologie die Krisis des Zeitalters überwinden könne, indem sie sie durchschaut, daß Politik eines Tages angewandte Soziologie sein werde, wie Technik angewandte Physik ist ${ }^{\prime 134}$. Denn nur der Soziologe ist nach Freyer dazu in der Lage, die in der Gegenwart vorhandenen Veränderungstendenzen richtig einzuschätzen, die richtige als zentral wirksam zu definieren und dann in wissenschaftlicher Weise zu ihrer Realisierung beizutragen 135 . Auch Karl Mannheim sah den Ausweg aus der Fragmentierung der politischen und wissenschaftlichen Kultur in seiner Wissenssoziologie und der mit ihr verbundenen Theorie der Syntheseleistung der freischwebenden Intelligenz, für die er zweifelsohne selbst das beste Beispiel war' ${ }^{136}$. Im Unterschied zu den bisher Zitierten außerhalb der etablierten Wissenschaften stehend, sah auch Ernst Bloch seine Werke als einen Versuch, den Geist der Utopie zu stärken und so zu deren Verwirklichung beizutragen ${ }^{137}$.

\section{Gestaltbarkeitsbewußtsein-Relevanz und Akzeptanz der Utopie}

Das Verständnis der Utopie als gesellschaftsgestaltende Kraft und das Bewußtsein der Diskursteilnehmenden, Veränderungen zu bewirken, reflektierten das seit dem ausgehenden 19. Jahrhundert im Zuge der rasanten gesellschaftlichen, wirtschaftlichen und technischen Veränderungsprozesse gewachsene Gestaltbarkeitsbewußtsein ${ }^{138}$ und die damit einhergegangene Akzeptanz- und Relevanzsteige-

131 Siehe zu diesem Selbstverständnis auch: Volker Kruse, Soziologie und "Gegenwartskrise“. Die Zeitdiagnosen Franz Oppenheimers und Alfred Webers (Wiesbaden 1990) bes. 30-34.

132 Landauer, Revolution 10.

133 S. o. Anm. 51.

134 Freyer, Soziologie 2.

135 Ebd. 206, 295-307. Konkret bedeutete dies für Freyer, die Revolution von rechts zu fördern.

136 S. o. Anm. 119.

137 S. o. Anm. 59.

138 Grundsätzlich kann dieser Prozeß als Radikalisierung des von Reinhart Koselleck für die „Sattelzeit ${ }^{\star}$ beschriebenen Auseinandertretens von Erfahrungsraum und Erwartungshorizont gedacht werden. Dabei ist der zentrale Aspekt jedoch nicht, daß die Erwartung als nicht mehr aus der Erfahrung ableitbar erscheint, sondern vielmehr, daß die Zukunft als offener Raum menschlicher Gestaltung begriffen wird. Reinhart Koselleck, Erfahrungsraum und Erwartungshorizont - zwei historische Kategorien, in: ders., Vergangene Zukunft. Zur Semantik geschichtlicher Zeiten (Frankfurt a.M. ${ }^{3} 1995$ ) 349-375. Dazu: Sighard Neckel, 
rung des utopischen Denkens. Dieser letzte Kontext, der die Entstehung des sozialwissenschaftlichen Utopiediskurses beeinflußte, ist von einem solchen Abstraktionsgrad, daß er nur indirekt erschlossen werden kann.

Den vielleicht deutlichsten Indikator für das wachsende Bewußtsein der Kontingenz und Gestaltbarkeit menschlicher Lebensverhältnisse bildet die Intensivierung der gesellschaftlichen und wirtschaftlichen Planungsdiskurse: Im Verlauf der Etablierung der Sozial- und Humanwissenschaften ${ }^{139}$ beanspruchte ein neuer Typus des "humanwissenschaftlichen Experten “ immer mehr Gesellschaftsgestaltungskompetenz ${ }^{140}$. Darüber hinaus äußerte sich das wachsende Gestaltbarkeitsbewußtsein in der Ausweitung der radikalen Lebensreformdiskurse sowie der Gründung der diversen Lebensreformverbände ${ }^{141}$. Eine wesentliche Plausibilisierung erfuhren die verschiedenen Diskurse über die zukünftige Gestaltung der $\mathrm{Ge}$ sellschaftsordnung durch die schnellen technischen Innovationen, die sich im gleichen Zeitraum vollzogen. So bemerkte bereits Werner Sombart: „Wenn sich so viel geändert hat, wenn solche Wunder, an die niemand je zu glauben gewagt hatte, sich spielend vor unseren Augen verwirklichen: warum nicht noch mehr? Warum nicht alles Wünschbare? So wird die revolutionäre Gegenwart zum Nährboden für die soziale Utopie der Zukunft. Edison und Siemens sind die Väter von Bellamy und Bebel. ${ }^{\text {142 }}$ Daher diente die Analogisierung von technischer und gesellschaftstechnischer Konstruktion häufig als Argument für die Realisierbarkeit utopischer Gesellschaftsentwürfe.

Über den allgemeinen Kontext des wachsenden Gestaltbarkeitsbewußtseins kann auch ein Ansatz zur Erklärung der Intensivierung des sozialwissenschaftlichen Utopiediskurses in der Weimarer Republik gefunden werden: Die radikalen und teilweise geplanten wirtschaftlichen und gesellschaftlichen Veränderungsprozesse im Verlauf des Ersten Weltkriegs sowie die Russische Revolution ${ }^{143}$ und die

Entzauberung der Zukunft. Zur Geschichte und Theorie sozialer Zeitperspektiven, in: Zerstörung und Wiederaneignung von Zeit, hrsg. v. Rainer Zoll (Frankfurt a.M. 1988) 464-486, bes. 474 .

139 Vgl. die inzwischen klassischen Untersuchungen von Detlev J. K. Peukert, Die Weimarer Republik. Krisenjahre der klassischen Moderne (Frankfurt a. M. 1987) bes. 137-139; ders., Die Genesis der Endlösung aus dem Geist der Wissenschaft, in: Zerstörung des moralischen Selbstbewußtseins: Chance oder Gefährdung? Praktische Philosophie in Deutschland nach dem Nationalsozialismus hrsg. v. Siegfried Blaschke u. a. (Frankfurt a.M. 1988) 24-48, bes. 28-33.

140 Zum Begriff der "humanwissenschaftlichen Experten“ aus Medizin, Eugenik, Sozialhygiene, Psychologie, Kriminologie, Pädagogik und Soziologie und ihrer Rolle im 20. Jahrhundert: Lutz Raphael, Die Verwissenschaftlichung des Sozialen als methodische und konzeptionelle Herausforderung für eine Sozialgeschichte des 20. Jahrhunderts, in: GG 22 (1996) 165-193.

141 Vgl. einführend: Diethard Kerbs (Hrsg.), Handbuch der deutschen Reformbewegungen, 1880-1933 (Wuppertal 1998).

142 Werner Sombart, Sozialismus und soziale Bewegung im 19. Jahrhunder (Jena 61908) 9. $\mathrm{Vgl}$. dazu auch in bezug auf die Produktion von Utopien: Hölscher, Entdeckung 130f., 152-154.

143 Vgl. Donal O'Sullivan, Furcht und Faszination - Deutsche und britische Rußlandbilder 1921-1933 (Köln, Weimar, Wien 1996). Zu Ausmaß und Intensität der intellektuellen Rezep- 
revolutionären Bewegungen in den europäischen Ländern erhöhten das allgemeine Bewußtsein der Zeitgenossen für die Kontingenz und Gestaltbarkeit gesellschaftlicher Lebensverhältnisse und damit die Akzeptanz der Utopien ${ }^{144}$. Darüber hinaus stieg nach dem Ende des Kaiserreiches und dem Scheitern der kommunistischen Revolutionsversuche in Deutschland der Utopiebedarf sowohl auf der politischen Linken als auch auf der politischen Rechten - im Spektrum des konservativen Utopismus - an. Die direkte Teilnahme an Krieg oder Revolution oder zumindest die enge Verbindung mit diesen Ereignissen übte für die meisten Utopietheoretiker großen Einfluß auf ihre Reflexionen aus ${ }^{145}$ : Beeindruckt durch die Erfahrung der Gestaltbarkeit menschlicher Lebensverhältnisse sowie die Beobachtung der revolutionären Bewegungen, diente ihnen der Utopiebegriff dazu, das geistige Moment in diesen Transformationsprozessen zu erfassen. Wie es sich für Intellektuelle gehört, erhoben sie es dabei zum zentralen movens der Veränderungsprozesse. Somit wiesen sie nicht zuletzt sich selbst als Vertretern des Geistigen die zentrale Rolle für die Interpretation und Organisation der gesellschaftlichen Transformationsprozesse zu, die aufgrund der allgemeinen Krisenwahrnehmung als notwendig erachtet wurden. In diesem Sinne reflektierte die Herausbildung des sozialpsychologischen Utopiediskurses auch das Selbstverständnis von Sozialwissenschaftlern und politischen Intellektuellen vor dem Hintergrund der intensiven Krisenerfahrungen des beginnenden 20. Jahrhunderts.

tion der russischen Revolution in Deutschland siehe die Bibliographie der zeitgenössischen Literatur in: Gerd Koenen, Lew Kopelew (Hrsg.), Deutschland und die Russische Revolution 1917-1924 (München 1998) 836-917. Als Beispiel siehe: Heinrich v. Gleichen, Annalise Schmidt. Der Bolschewismus und die deutschen Intellektuellen. Äußerungen auf eine Umfrage des Bundes deutscher Gelehrter und Künstler (Leipzig 1920).

144 Vgl.: Peter Frizzsche, Landscape of Danger, Landscape of Design. Crisis and Modernism in Weimar Germany, in: Dancing on the Volcano. Essays on the Culture of the Weimar Republic, hrsg. v. Thomas W. Kniesche, Stephen Brockmann (Columbia 1994) 29-46, 40: „The war and the revolutions of 1917 and 1918 confirmed the vastness of possibility, an open space and threatened conventional approaches and traditional orders." Siehe auch: Peter Krüger, Der Erste Weltkrieg als Epochenschwelle, in: Wege in die Gewalt. Die modernen politischen Religionen, hrsg. v. Hans Maier (Frankfurt a. M. 2000) 70-91. Weltkrieg und Revolution waren von internationaler Bedeutung für den Utopiediskurs. Vgl.: Lewis Mumford, Sketches from Life. The Early Years (Boston 1982) $241 \mathrm{f} ., 303 \mathrm{f}$.

145 Gustav Landauer und Otto Neurath engagierten sich in der Münchener Räterepublik, vgl. Braun, Utopie 50; und Anm. 50. Ernst Bloch wandte sich im Schweizer Exil publizistisch gegen den Krieg und begrüßte die Russische Revolution, vgl. Peter Zudeick, Der Hintern des Teufels. Ernst Bloch. Leben und Werk (Moos/Baden Baden 1985) 71-80. Hans Freyer kämpfte als Leutnant im Weltkrieg und kehrte zurück in die revolutionären Wirren in Leipzig, vgl. Muller, The Other God 58-74. Karl Mannheim nahm - vermittelt durch Georg Lukács - Anteil an den Entwicklungen der ungarischen Räterepublik, vgl. Kettler, Culture 37 und passim. 
